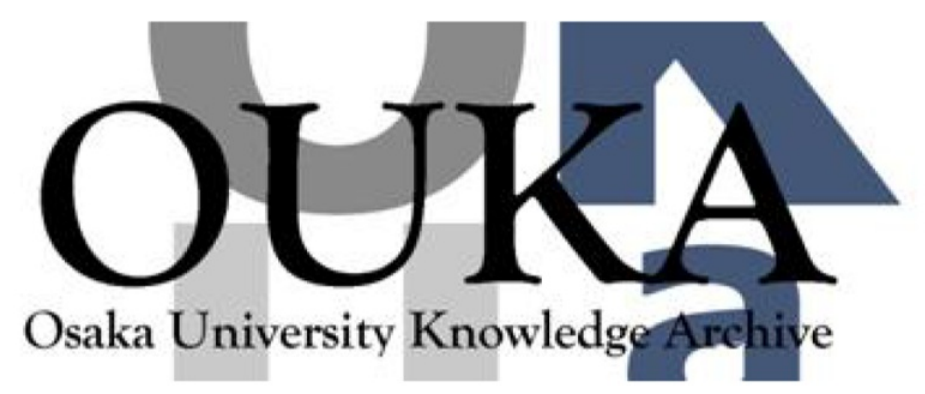

\begin{tabular}{|c|l|}
\hline Title & $\begin{array}{l}\text { Rotating black holes at future colliders. III. } \\
\text { Determination of black hole evolution }\end{array}$ \\
\hline Author(s) & Ida, Daisuke; Oda, Kinya; Park, Seong Chan \\
\hline Citation & Physical Review D. 73(12) p. 124022 \\
\hline Issue Date & $2006-06-15$ \\
\hline oaire:version & VoR \\
\hline URL & https://hdl.handle.net/11094/78772 \\
\hline rights & ○ 2006 American Physical Society \\
\hline Note & \\
\hline
\end{tabular}

Osaka University Knowledge Archive : OUKA

https://ir. Library. osaka-u. ac. jp/

0saka University 


\title{
Rotating black holes at future colliders. III. Determination of black hole evolution
}

\author{
Daisuke Ida, ${ }^{1}$ Kin-ya Oda, ${ }^{2}$ and Seong Chan Park ${ }^{3}$ \\ ${ }^{1}$ Department of Physics, Gakushuin University, Tokyo 171-8588, Japan \\ ${ }^{2}$ Institute of Theoretical Physics, Warsaw University, Hoża 69, Warsaw 00-681, Poland \\ ${ }^{3}$ LEPP, Cornell University, Ithaca, New York 14853, USA \\ (Received 28 February 2006; published 15 June 2006)
}

\begin{abstract}
$\mathrm{TeV}$ scale gravity scenario predicts that the black hole production dominates over all other interactions above the scale and that the Large Hadron Collider will be a black hole factory. Such higher-dimensional black holes mainly decay into the standard model fields via the Hawking radiation whose spectrum can be computed from the greybody factor. Here we complete the series of our work by showing the greybody factors and the resultant spectra for the brane-localized spinor and vector field emissions for arbitrary frequencies. Combining these results with the previous works, we determine the complete radiation spectra and the subsequent time evolution of the black hole. We find that, for a typical event, well more than half a black hole mass is emitted when the hole is still highly rotating, confirming our previous claim that it is important to take into account the angular momentum of black holes.
\end{abstract}

DOI: 10.1103/PhysRevD.73.124022

PACS numbers: 04.70.Dy, 04.50.+h, 11.25.Mj, 11.25.Wx

\section{INTRODUCTION}

Black holes have been playing crucial roles for decades in the yet unaccomplished theoretical development to reconcile gravitational interactions with quantum description of nature [1-7]. From this point of view, it is no doubt desirable to have a direct experimental test of the Hawking radiation [2] through which black holes are expected to radiate off quanta almost thermally. An astrophysical black hole is too big to have a sizable Hawking temperature for this purpose. It has long been known that a collision of two particles above the Planck energy scale inevitably leads to a production of a black hole [8], which can have large Hawking temperature.

Until recently, the Planck scale was always assumed to be unaccessibly high for human beings even in theories with extra compact dimensions such as string theory, under the assumption that the compactification radius is of the order of the Planck length. Without prejudice, there is no reason that forces one to keep this assumption. Recently it has been pointed out that the large extra dimensions can reduce the higher-dimensional Planck scale down to $\mathrm{TeV}$ scale to solve the so-called hierarchy problem without contradicting any experimental data if the standard model interactions are confined to a 3-brane [9-11]. It also has been pointed out that a $\mathrm{TeV}$ scale gravity is realized if an extra dimension is compactified with the warped metric on $\mathrm{AdS}_{5}$ [12].

The TeV scale gravity opens up a possibility of producing black holes and observing their decay products directly at a next generation collider. First it was considered that such a black hole will mainly decay into the bulk graviton modes $[13,14]$. Later it has been noticed that the decay channels into observable brane-localized standard model fields dominate over the bulk graviton emission [15]. When the black hole is highly rotating, the bulk graviton emission is expected to be greatly enhanced [16-19]. Currently the bulk graviton equation is obtained only for the nonrotating $J=0$ hole [20-22] and we cannot conclude how much the graviton emission will be enhanced. There is also an argument that the higher-dimensional graviton has a larger number of modes than four dimensions and that the graviton emission would be enhanced due to this fact $[16,17]$. The actual number of modes that have smaller masses than the Hawking temperature, which is always smaller than the higher-dimensional Planck scale, will depend on the details of the moduli stabilization. Typically one has much fewer light modes after the moduli fixing. The possibility of black hole production at collider and the detection of its Hawking radiation is studied from a general perspective in [23] and the experimental signature at the CERN Large Hadron Collider is studied in [24] in the approximation that the black hole has vanishing anglar momentum $J=0$ and that the Hawking radiation spectrum is described with the high frequency limit $r_{h} \omega \gg 1$, where $r_{h}$ and $\omega$ are the black hole horizon radius and the energy of the emitted particle, respectively.

In [25], we have emphasized the importance to take into account the angular momentum of the black hole and have first estimated the differential production cross section for a given $J$, whose integral over $J$ qualitatively agrees with the numerical results of the classical gravitational collision of two massless particles [26,27], namely, the fact that the total cross section increases from the Schwarzschild approximation more and more for higher dimensions is well described. ${ }^{1}$ Further, utilizing the Newman-Penrose formalism [30] we have obtained the field equations of the branelocalized scalar $(s=0)$, spinor $(s=1 / 2)$, and vector $(s=$ 1) fields in a separable form for the $D=4+$

\footnotetext{
${ }^{1}$ A similar formula has appeared in [28], which predicts that the cross section would be reduced by taking into account the angular momentum, contrary to our result. See also [29] for another earlier attempt.
} 
$n$-dimensional black hole background with a single angular momentum $J \geq 0$ [31]. Later, these equations are also derived for a nonrotating $J=0$ black hole in Refs. [32,33].

Once shown to be separable, its radial part can be solved to obtain the greybody factors that completely determine the Hawking radiation spectra without relying on the high frequency limit $r_{h} \omega \gg 1$. A thermal radiation spectrum at the horizon is modified when the radiation passes through the curved geometry toward an observer located at the spatial infinity. The greybody factor is introduced to take this correction into account for each angular mode $(l, m)$ and frequency $\omega$ of the emitted radiation. It is defined to be the absorption rate by the hole for a steady in-falling flux at the infinity

$$
\Gamma_{l m}=\frac{\dot{N}_{l m}^{(\text {in })}-\dot{N}_{l m}^{(\text {out })}}{\dot{N}_{l m}^{(\text {(in) }}}
$$

under the condition that the radial wave is purely ingoing at the horizon. This definition physically means, in a timereversed sense, that $\Gamma$ is identical to the proportion that passes through the gravitational potential from the horizon toward the infinity without being reflected back.

In the early 1970's, Teukolsky showed that the general wave equation in the four-dimensional Kerr black hole background is separable into the angular and radial parts and developed analytic and numerical methods to solve the equation [34-36]. Page then calculated the Hawking radiation spectra with the greybody factors taken into account [37] and showed the whole time evolution of the four-dimensional Kerr black hole [38]. The brane field equations that we have obtained in [25] is a generalization of the Teukolsky equation. In [25], we have further obtained the analytic formulas of the greybody factors of the $s=0,1 / 2$ and 1 brane fields in the low frequency limit $r_{h} \omega \ll 1$ for $D=5$-dimensional $J \geq 0$ black hole.

Our next goal is to obtain the greybody factors without relying on the low frequency expansions. The numerical results with nonrotating $J=0$ black holes are shown by Harris and Kanti in [33]. The greybody factors of $s=0$ brane field for general $J \geq 0$ hole are presented by ourselves $[39,40]$ and by Harris and Kanti $[41,42]$. Recently, there also appeared a paper that treats the $s=1$ modes for $J \geq 0$ black hole in a restricted range $r_{h} \omega<4$ [43].

In this paper, we complete the series of our work by presenting the greybody factors for $s=1 / 2$ and $s=1$ brane fields for the general $J \geq 0$ hole and the resultant Hawking spectra. Now that all the brane field spectra are completed, we are finally able to show the whole time evolution of the black hole to confirm our previous claim that the spin-down phase, where the approximation $J=0$ is not valid, is hardly negligible: we find that typically much more than a half of the black hole mass is emitted in the spin-down phase.

The organization of the paper is as follows: In Sec. II, we go beyond our previous treatment to obtain the brane field equations in a separable form for the lower spin components too and recast the results into rather simple formulas (51) and (52). We then show the resultant forms of the asymptotic solutions and the greybody factors. In Sec. III, we explain our numerical methods to evade the contamination of the outgoing wave near the horizon when we impose the purely ingoing boundary condition there. In Sec. IV, we show our results of the greybody factors and the Hawking spectra for the brane-localized spinor and vector fields. In Sec. V, we combine all the results of scalar [40] and spinor/vector obtained in the previous section to determine the whole time evolution of the black hole. The time evolution for the Randall-Sundrum $(D=5)$ and the Arkani-Hamed, Dimopoulos, and Dvali (ADD) $(D=10)$ black hole are presented.

\section{BRANE FIELD EQUATIONS}

The stationary rotating black holes would be described by the Myers-Perry solution [31] as indicated in [44-49]. The induced metric on the three-brane in the $(4+$ $n$ )-dimensional Myers-Perry solution with a single nonzero angular momentum is given by

$$
\begin{aligned}
g= & \frac{\Delta-a^{2} \sin ^{2} \vartheta}{\Sigma} d t^{2}+\frac{2 a\left(r^{2}+a^{2}-\Delta\right) \sin ^{2} \vartheta}{\Sigma} d t d \varphi \\
& -\frac{\left(r^{2}+a^{2}\right)^{2}-\Delta a^{2} \sin ^{2} \vartheta}{\Sigma} \sin ^{2} \vartheta d \varphi^{2} \\
& -\frac{\Sigma}{\Delta} d r^{2}-\Sigma d \vartheta^{2},
\end{aligned}
$$

where

$$
\Sigma=r^{2}+a^{2} \cos ^{2} \vartheta, \quad \Delta=r^{2}+a^{2}-\mu r^{1-n} .
$$

The parameters $\mu$ and $a$ are equivalent to the total mass $M$ and the angular momentum $J$

$$
M=\frac{(2+n) A_{2+n} \mu}{16 \pi G_{4+n}}, \quad J=\frac{A_{2+n} \mu a}{8 \pi G_{4+n}}
$$

evaluated at the spatial infinity of the $(4+n)$-dimensional space-time, respectively, where $A_{2+n}=2 \pi^{(3+n) / 2} / \Gamma((3+$ $n) / 2)$ is the area of a unit $(2+n)$ sphere and $G_{4+n}$ is the $(4+n)$-dimensional Newton constant of gravitation.

In Ref. [25] it has been shown in terms of the NewmanPenrose formalism that the spin $s=0,1 / 2$ and 1 equations are of separable type in the background space-time with the metric (2). Here we give the basic equations in more compactified notation. 
The null tetrad $\left\{n, n^{\prime}, m, m^{\prime}\right\}$ on the brane is

$$
\begin{aligned}
n= & \partial_{t}-a \sin ^{2} \vartheta \partial_{\varphi}-\frac{\Sigma}{\Delta} \partial_{r}, \\
n^{\prime}= & \frac{\Delta}{2 \Sigma}\left(\partial_{t}-a \sin ^{2} \vartheta \partial_{\varphi}\right)+\frac{1}{2} \partial_{r}, \\
m= & \frac{i \sin \vartheta}{\sqrt{2}(r+i a \cos \vartheta)}\left[a \partial_{t}-\left(r^{2}+a^{2}\right) \partial_{\varphi}\right] \\
& -\frac{r-i a \cos \vartheta}{\sqrt{2}} \partial_{\vartheta}, \\
m^{\prime}= & \bar{m} .
\end{aligned}
$$

The spin coefficients [30] in this tetrad system become

$$
\begin{array}{ll}
\tau=-\frac{i a \sin \vartheta}{\sqrt{2} \Sigma}, & \rho=-\frac{1}{r-i a \cos \vartheta}, \\
\beta=-\frac{\bar{\rho} \cot \vartheta}{2 \sqrt{2}}, & \tau^{\prime}=-\frac{i a \rho^{2} \sin \vartheta}{\sqrt{2}}, \\
\rho^{\prime}=-\frac{\rho^{2} \bar{\rho} \Delta}{2}, & \epsilon^{\prime}=\rho^{\prime}-\frac{\rho \bar{\rho} \Delta_{, r}}{4}, \\
\beta^{\prime}=\tau^{\prime}+\bar{\beta}, & \kappa=\sigma=\kappa^{\prime}=\sigma^{\prime}=\epsilon=0 .
\end{array}
$$

The null tetrad basis vectors define the differential operators

$$
\left(D, D^{\prime}, m, m^{\prime}\right)=\left(\nabla_{n}, \nabla_{n^{\prime}}, \nabla_{m}, \nabla_{m^{\prime}}\right) .
$$

In terms of these differential operators and the spincoefficients, the field equations for $s=1 / 2$ fields $\left(\psi_{0}, \psi_{1}\right)$ (Weyl equation) are given by

$$
\begin{aligned}
& D \psi_{1}-\delta^{\prime} \psi_{0}=\left(\beta^{\prime}-\tau^{\prime}\right) \psi_{0}+(\rho-\epsilon) \psi_{1}, \\
& \delta \psi_{1}-D^{\prime} \psi_{0}=\left(\epsilon^{\prime}-\rho^{\prime}\right) \psi_{0}+(\tau-\beta) \psi_{1} .
\end{aligned}
$$

On the other hand, the field equations for $s=1$ fields $\left(\phi_{0}, \phi_{1}, \phi_{2}\right)$ (Maxwell equation) are given by

$$
\begin{gathered}
D \phi_{1}-\delta^{\prime} \phi_{0}=\left(2 \beta^{\prime}-\tau^{\prime}\right) \phi_{0}+2 \rho \phi_{1}-\kappa \phi_{2}, \\
D \phi_{2}-\delta^{\prime} \phi_{1}=\sigma^{\prime} \phi_{0}-2 \tau^{\prime} \phi_{1}+(\rho-2 \epsilon) \phi_{2}, \\
D^{\prime} \phi_{0}-\delta \phi_{1}=\left(\rho^{\prime}-2 \epsilon^{\prime}\right) \phi_{0}-2 \tau \phi_{1}+\sigma \phi_{2}, \\
D^{\prime} \phi_{1}-\delta \phi_{2}=-\kappa^{\prime} \phi_{0}+2 \rho^{\prime} \phi_{1}+(2 \beta-\tau) \phi_{2} .
\end{gathered}
$$

The all field variables are assumed to have time and angular dependence $\phi_{A}, \psi_{A} \propto e^{-i \omega t+i m \varphi}$.

Here it is useful to introduce the differential operators

$$
\begin{aligned}
\mathcal{D} & =\partial_{r}-i \frac{K}{\Delta}, \\
\mathcal{D}^{\dagger} & =\partial_{r}+i \frac{K}{\Delta}, \\
\mathcal{L}_{N} & =\partial_{\vartheta}+Q+N \cot \vartheta, \\
\mathcal{L}_{N}^{\dagger} & =\partial_{\vartheta}-Q+N \cot \vartheta, \\
(N & =0, \pm 1 / 2, \pm 1),
\end{aligned}
$$

where

$$
\begin{aligned}
& K(r)=\omega\left(r^{2}+a^{2}\right)-m a, \\
& Q(\vartheta)=-\omega a \sin \vartheta+m \csc \vartheta
\end{aligned}
$$

have been defined.

\section{A. $s=1 / 2$ field equations}

The $s=1 / 2$ field Eqs. (7) and (8) can be written as

$$
\begin{aligned}
& \mathcal{D} \Delta^{1 / 2} \eta_{-1 / 2}=-\mathcal{L}_{1 / 2} \eta_{1 / 2}, \\
& \mathcal{D}^{\dagger} \Delta^{1 / 2} \eta_{1 / 2}=\mathcal{L}_{1 / 2}^{\dagger} \eta_{-1 / 2},
\end{aligned}
$$

where

$$
\eta_{-1 / 2}=\sqrt{2} \Delta^{-1 / 2} \rho^{-1} \psi_{1}, \quad \eta_{1 / 2}=\psi_{0}
$$

have been defined. From $\mathcal{L}_{1 / 2}^{\dagger} \times$ Eq. (15) $+\mathcal{D} \Delta^{1 / 2} \times$ Eq. (16) we obtain

$$
\mathcal{D} \Delta^{1 / 2} \mathcal{D}^{\dagger} \Delta^{1 / 2} \eta_{1 / 2}=-\mathcal{L}_{1 / 2}^{\dagger} \mathcal{L}_{1 / 2} \eta_{1 / 2} .
$$

By putting $\eta_{1 / 2}=R_{1 / 2}(r) S_{1 / 2}(\vartheta) e^{-i \omega t+i m \varphi}$, we have separated equations

$$
\begin{gathered}
\mathcal{D} \Delta^{1 / 2} \mathcal{D}^{\dagger} \Delta^{1 / 2} R_{1 / 2}=\lambda_{1 / 2} R_{1 / 2}, \\
\mathcal{L}_{1 / 2}^{\dagger} \mathcal{L}_{1 / 2} S_{1 / 2}=-\lambda_{1 / 2} S_{1 / 2},
\end{gathered}
$$

where $\lambda_{1 / 2}$ is the separation constant. In a similar manner, $\mathcal{D}^{\dagger} \Delta^{1 / 2} \times(15)-\mathcal{L}_{1 / 2} \times(16)$ and the substitution $\eta_{-1 / 2}=$ $R_{-1 / 2}(r) S_{-1 / 2}(\vartheta) e^{-i \omega t+i m \varphi}$ give the separated equations

$$
\begin{gathered}
\mathcal{D}^{\dagger} \Delta^{1 / 2} \mathcal{D} \Delta^{1 / 2} R_{-1 / 2}=\lambda_{1 / 2} R_{-1 / 2}, \\
\mathcal{L}_{1 / 2} \mathcal{L}_{1 / 2}^{\dagger} S_{-1 / 2}=-\lambda_{1 / 2} S_{-1 / 2} .
\end{gathered}
$$

The separation constant must again be $\lambda_{1 / 2}$, which can be seen from explicit forms of angular Eqs. (20) and (22). Hereafter, the angular function $S_{A}(\vartheta)$ is normalized such that

$$
\int_{0}^{\pi}\left|S_{A}(\vartheta)\right|^{2} \sin \vartheta d \vartheta=\frac{1}{2 \pi} .
$$

Furthermore, from Eqs. (15) and (16), we obtain relationships

$$
\begin{gathered}
S_{-1 / 2}=-\frac{1}{\sqrt{\lambda_{1 / 2}}} \mathcal{L}_{1 / 2} S_{1 / 2}, \\
S_{1 / 2}=\frac{1}{\sqrt{\lambda_{1 / 2}}} \mathcal{L}_{1 / 2}^{\dagger} S_{-1 / 2},
\end{gathered}
$$

and

$$
\mathcal{D} \Delta^{1 / 2} R_{-1 / 2}=\sqrt{\lambda_{1 / 2}} R_{1 / 2},
$$


DAISUKE IDA, KIN-YA ODA, AND SEONG CHAN PARK

$$
\mathcal{D}^{\dagger} \Delta^{1 / 2} R_{1 / 2}=\sqrt{\lambda_{1 / 2}} R_{-1 / 2},
$$

where the constant of proportionality can be determined by noting that

$$
\begin{aligned}
\left(\mathcal{L}_{1 / 2} S_{1 / 2}\right)^{2} \sin \vartheta= & \lambda_{1 / 2} S_{1 / 2}^{2} \sin \vartheta+\partial_{\vartheta}\left(S_{1 / 2} \mathcal{L}_{1 / 2} S_{1 / 2}\right) \\
\left(\mathcal{L}_{1 / 2}^{\dagger} S_{-1 / 2}\right)^{2} \sin \vartheta= & \lambda_{1 / 2} S_{-1 / 2}^{2} \sin \vartheta \\
& +\partial_{\vartheta}\left(S_{-1 / 2} \sin \vartheta \mathcal{L}_{1 / 2}^{\dagger} S_{-1 / 2}\right)
\end{aligned}
$$

hold.

\section{B. $s=1$ field equations}

The $s=1$ field Eqs. (9)-(12) become

$$
\begin{gathered}
\mathcal{D} \eta=-\rho^{-2} \mathcal{L}_{1} \rho \eta_{1}, \\
\mathcal{L}_{0} \eta=-\rho^{-2} \mathcal{D} \rho \Delta \eta_{-1}, \\
\mathcal{L}_{0}^{\dagger} \eta=\rho^{-2} \mathcal{D}^{\dagger} \rho \Delta \eta_{1}, \\
\mathcal{D}^{\dagger} \eta=\rho^{-2} \mathcal{L}_{1}^{\dagger} \rho \eta_{-1},
\end{gathered}
$$

where

$$
\eta_{1}=\phi_{0}, \quad \eta_{-1}=2 \Delta^{-1} \rho^{-2} \phi_{2}, \quad \eta=\sqrt{2} \rho^{-2} \phi_{1}
$$

have been defined. From $\mathcal{L}_{0}^{\dagger} \times$ Eq. (30) $-\mathcal{D} \times$ Eq. (32), we obtain

$$
\begin{aligned}
& {\left[(\mathcal{D}-\rho)\left(\mathcal{D}^{\dagger}+\rho\right) \Delta+\left(\mathcal{L}_{0}^{\dagger}-i \rho a \sin \vartheta\right)\left(\mathcal{L}_{1}+i \rho a \sin \vartheta\right)\right] \eta_{1}} \\
& \quad=\left(\mathcal{D D}^{\dagger} \Delta+\mathcal{L}_{0}^{\dagger} \mathcal{L}_{1}-\frac{2 i \omega}{\bar{\rho}}\right) \eta_{1}=0
\end{aligned}
$$

Putting $\eta_{1}=R_{1}(r) S_{1}(\vartheta) e^{-i \omega t+i m \varphi}$, we obtain separated equations

$$
\begin{gathered}
\left(\mathcal{D} \mathcal{D}^{\dagger} \Delta+2 i \omega r\right) R_{1}=\lambda_{1} R_{1}, \\
\left(\mathcal{L}_{0}^{\dagger} \mathcal{L}_{1}-2 \omega a \cos \vartheta\right) S_{1}=-\lambda_{1} S_{1},
\end{gathered}
$$

where $\lambda_{1}$ is the separation constant. In a similar manner, from $\mathcal{D}^{\dagger} \times$ Eq. (31) $-\mathcal{L}_{0} \times$ Eq. (33) we obtain

$$
\begin{gathered}
{\left[\left(\mathcal{D}^{\dagger}-\rho\right)(\mathcal{D}+\rho) \Delta+\left(\mathcal{L}_{0}-i \rho a \sin \vartheta\right)\left(\mathcal{L}_{1}^{\dagger}+i \rho a \sin \vartheta\right)\right] \eta_{-1}} \\
=\left(\mathcal{D}^{\dagger} \mathcal{D} \Delta+\mathcal{L}_{0} \mathcal{L}_{1}^{\dagger}+\frac{2 i \omega}{\bar{\rho}}\right) \eta_{-1} .
\end{gathered}
$$

Putting $\eta_{-1}=R_{-1}(r) S_{-1}(\vartheta) e^{-i \omega t+i m \varphi}$, we obtain another set of separated equations

$$
\begin{gathered}
\left(\mathcal{D}^{\dagger} \mathcal{D} \Delta-2 i \omega r\right) R_{-1}=\lambda_{1} R_{-1}, \\
\left(\mathcal{L}_{0} \mathcal{L}_{1}^{\dagger}+2 \omega a \cos \vartheta\right) S_{-1}=-\lambda_{1} S_{-1}
\end{gathered}
$$

PHYSICAL REVIEW D 73, 124022 (2006)

The constant of separation $\lambda_{1}$ is again common for both sets of equations.

The relationship between $\eta_{1}$ and $\eta_{-1}$ is seen as follows: From $\mathcal{L}_{0} \times$ Eq. (30) $-\mathcal{D} \times$ Eq. (31), we obtain

$$
\mathcal{D} \mathcal{D} \Delta \eta_{-1}=\mathcal{L}_{0} \mathcal{L}_{1} \eta_{1}
$$

From $\mathcal{D}^{\dagger} \times$ Eq. (32) $-\mathcal{L}_{0}^{\dagger} \times$ Eq. (33), we obtain

$$
\mathcal{D}^{\dagger} \mathcal{D}^{\dagger} \Delta \eta_{1}=\mathcal{L}_{0}^{\dagger} \mathcal{L}_{1}^{\dagger} \eta_{-1} .
$$

These imply that

$$
\begin{gathered}
\mathcal{L}_{0} \mathcal{L}_{1} S_{1}=B S_{-1}, \\
\mathcal{D} \mathcal{D} \Delta R_{-1}=B R_{1}, \\
\mathcal{L}_{0}^{\dagger} \mathcal{L}_{1}^{\dagger} S_{-1}=B S_{1}, \\
\mathcal{D}^{\dagger} \mathcal{D}^{\dagger} \Delta R_{1}=B R_{-1},
\end{gathered}
$$

where $B$ is a constant. The constant $B$ is determined by

$$
\begin{aligned}
B^{2} & =\int\left(\mathcal{L}_{0} \mathcal{L}_{1} S_{1}\right)^{2} \sin \vartheta d \vartheta=\int S_{1} \mathcal{L}_{0}^{\dagger} \mathcal{L}_{1}^{\dagger} \mathcal{L}_{0} \mathcal{L}_{1} S_{1} \\
& =\lambda_{1}^{2}-4 \omega a(\omega a-m) .
\end{aligned}
$$

We choose

$$
B=\sqrt{\lambda_{1}^{2}-4 \omega a(\omega a-m)} .
$$

\section{Master equations for brane fields}

The angular and radial equations can be recasted into a neat form

$$
\begin{gathered}
{\left[\left(\partial_{\vartheta}-\frac{s}{|s|} Q+(1-|s|) \cot \vartheta\right)\left(\partial_{\vartheta}+\frac{s}{|s|} Q+|s| \cot \vartheta\right)\right.} \\
\left.+(2|s|-1) \frac{\left(\frac{s}{|s|} Q \sin \vartheta\right)_{, \vartheta}}{\sin \vartheta}+\lambda_{|s|}\right] S_{s}=0 \\
{\left[\left(\partial_{r}-i \frac{s}{|s|} \frac{K}{\Delta}\right) \Delta^{1-|s|}\left(\partial_{r}+i \frac{s}{|s|} \frac{K}{\Delta}\right) \Delta^{|s|}\right.} \\
\left.+(2|s|-1) i \frac{s}{|s|} K_{, r}-\lambda_{|s|}\right] R_{s}=0
\end{gathered}
$$

More explicit forms are given by

$$
\begin{aligned}
\frac{1}{\sin \vartheta} \frac{d}{d \vartheta}\left(\sin \vartheta \frac{d S_{s}(\vartheta)}{d \vartheta}\right)+\left[(s-\omega a \cos \vartheta)^{2}\right. \\
-(s \cot \vartheta+m \csc \vartheta)^{2}-\omega a(\omega a-2 m) \\
\left.-|s|(|s|+1)+\lambda_{|s|}\right] S_{s}(\vartheta)=0,
\end{aligned}
$$




$$
\begin{aligned}
\Delta^{-|s|} \frac{d}{d r}\left(\Delta^{1+|s|} \frac{d R_{s}(r)}{d r}\right) & +\left[\frac{K^{2}-i s K \Delta_{, r}}{\Delta}+2 i s K_{, r}\right. \\
& \left.+|s| \Delta_{, r r}-\lambda_{|s|}\right] R_{s}(r)=0 .
\end{aligned}
$$

These expressions are valid also for the massless scalar field by setting $s=0$.

Besides we have relationships between different radial components

$$
\left(\partial_{r}+i \frac{K}{\Delta}\right) \Delta^{1 / 2} R_{1 / 2}=\sqrt{\lambda_{1 / 2}} R_{-1 / 2}
$$

for spinor fields and

$$
\left(\partial_{r}+i \frac{K}{\Delta}\right)\left(\partial_{r}+i \frac{K}{\Delta}\right) \Delta R_{1}=\sqrt{\lambda_{1}^{2}-4 \omega a(\omega a-m)} R_{-1}
$$

for vector fields.

\section{Asymptotic solutions}

Asymptotic far-field solution to the Eq. (52) is

$$
\begin{aligned}
R_{s} \rightarrow & Y_{s}^{(\mathrm{in})} \frac{e^{-i \omega_{*} \xi_{*}}}{\xi^{1-s+|s|}}\left(1-i \frac{\lambda_{|s|}+s-|s|}{2 \omega \xi}\right) \\
& +Y_{s}^{\text {(out) }} \frac{e^{i \omega_{*} \xi_{*}}}{\xi^{1+s+|s|}}\left(1+i \frac{\lambda_{|s|}-s-|s|}{2 \omega \xi}\right),
\end{aligned}
$$

where we have introduced the new radial coordinate

$$
r_{\star}=\int^{r} \frac{K}{\omega \Delta},
$$

and the dimensionless parameters $\xi=r / r_{h}, \xi_{*}=r_{\star} / r_{h}$, $\omega_{*}=\omega r_{h}$, and $a_{*}=a / r_{h}$ have been defined.

Then, Eqs. (53) and (54) give the relationship between coefficients

$$
\begin{gathered}
Y_{-1 / 2}^{\text {(out) }}=i \frac{2 \omega_{*}}{\sqrt{\lambda_{1 / 2}}} Y_{1 / 2}^{\text {(out) }}, \\
Y_{-1}^{\text {(out) }}=-\frac{4 \omega_{*}^{2}}{\sqrt{\lambda_{1}^{2}-4 \omega_{*} a_{*}\left(\omega_{*} a_{*}-m\right)}} Y_{1}^{\text {(out) }} .
\end{gathered}
$$

\section{E. Greybody factors}

The greybody factor is given by the absorption coefficient for the wave equations. By virtue of the Eqs. (57) and (58), the absorption coefficient can be calculated solely by solving a single radial equation.

The number flux vector for $s=1 / 2$ is

$$
j^{\mu}=k\left(n^{\mu} \psi_{1} \bar{\psi}_{1}+n^{\prime \mu} \psi_{0} \bar{\psi}_{0}-m^{\mu} \psi_{1} \bar{\psi}_{0}-m^{\prime \mu} \psi_{0} \bar{\psi}_{1}\right),
$$

where $k$ is a constant. The radial component becomes asymptotically

$$
\begin{aligned}
j^{r} & =k\left(\left|\psi_{1}\right|^{2}-\frac{\Delta}{2 \Sigma}\left|\psi_{0}\right|^{2}\right)=k \frac{\Delta}{2 \Sigma}\left(\left|\eta_{-1 / 2}\right|^{2}-\left|\eta_{1 / 2}\right|^{2}\right) \\
& \rightarrow \frac{k}{2 \Sigma}\left(\left|Y_{-1 / 2}^{\text {(out) }}\right|^{2}\left|S_{-1 / 2}\right|^{2}-\left|Y_{1 / 2}^{\text {(in) }}\right|^{2}\left|S_{1 / 2}\right|^{2}\right)
\end{aligned}
$$

$(r \rightarrow+\infty)$.

Therefore, the absorption coefficient is given by

$$
\Gamma_{1 / 2}=1-\frac{\dot{N}_{1 / 2}^{\text {(out) }}}{\dot{N}_{1 / 2}^{\text {(in) }}}=1-\frac{4 \omega_{*}^{2}}{\lambda_{1 / 2}} \frac{\left|Y_{1 / 2}^{\text {(out) }}\right|^{2}}{\left|Y_{1 / 2}^{\text {(in) }}\right|^{2}},
$$

for spinor fields, where $\dot{N}_{1 / 2}^{\text {(in/out) }}$ denotes the total number flux. The last expression is determined by solving only $s=$ $1 / 2$ equation.

In a similar manner, we have the absorption coefficient

$$
\begin{aligned}
\Gamma_{1} & =1-\frac{\dot{N}_{1}^{(\text {out })}}{\dot{N}_{1}^{\text {(in) }}} \\
& =1-\frac{16 \omega_{*}^{4}}{\left[\lambda_{1}^{2}-4 \omega_{*} a_{*}\left(\omega_{*} a_{*}-m\right)\right]} \frac{\left|Y_{1}^{\text {(out) }}\right|^{2}}{\left|Y_{1}^{(\text {in })}\right|^{2}},
\end{aligned}
$$

for vector fields.

\section{NUMERICAL METHODS TO OBTAIN GREYBODY FACTORS}

Next, we explain our numerical methods. In this section, we take the unit

$$
r_{h}=1 \text {, }
$$

and always consider the case $s \geq 0$ unless otherwise stated. First, we switch from the Boyer-Lindquist frame (2) to the ingoing Kerr-Newman frame by

$$
\begin{gathered}
d v=d t+\frac{r^{2}+a^{2}}{\Delta} d r, \\
d \tilde{\varphi}=d \varphi+\frac{a d r}{\Delta} .
\end{gathered}
$$

The radial wave equation now becomes

$$
\frac{d^{2} R}{d r^{2}}+\eta \frac{d R}{d r}+\zeta R=0
$$

with

$$
\eta=-\frac{(s-1) \Delta^{\prime}+2 i K}{\Delta}, \quad \zeta=\frac{2 i \omega r(2 s-1)-\lambda}{\Delta}
$$

For the angular eigenvalue $\lambda$, we use the expansion of the form

$$
\lambda=\sum_{n} C_{n}(a \omega)^{n}
$$

presented in [50]. The coefficients $C_{n}$ are given for each 
angular mode $(l, m)$ and dumps quite fast, like a multiple of inversed factorials, as $l$ increases. We take up to $n=6$ terms. The accuracy of the approximation can be checked by the relative size of the last $n=6$ term to the sum when $a \omega$ is within the relevant region to the peak of the power spectrum, a posteriori. We have checked that the ratio is at most of order $0.1 \%$ for each relevant region.

The asymptotic solutions in the near-horizon (NH) and far-field $(\mathrm{FF})$ regions are obtained as [25]

$$
\begin{aligned}
& R^{\mathrm{NH}} \sim W_{\text {in }}+W_{\text {out }} e^{2 i k r_{*}} \Delta^{s}, \\
& R^{\mathrm{FF}} \sim Y_{\text {in }} r^{2 s-1}+Y_{\text {out }} e^{2 i k r_{*}} / r,
\end{aligned}
$$

where $r_{*}$ is the tortoise coordinate defined by $d r_{*} / d r=$ $\left(r^{2}+a^{2}\right) / \Delta$ and $r_{*} \rightarrow r$ for $r \rightarrow \infty$. Recall that $\Delta \rightarrow 0$ for $r \rightarrow 1$. This coordinate change is not essential for the following analysis but convenient because the near-horizon ingoing solution does not contain the tortoise coordinate $r_{*}$.

\section{A. Removing outgoing contamination at near horizon}

For the case of scalar $(s=0)$ [40], the calculation is simply to put the purely ingoing boundary condition $W_{\text {in }}=$ 0 at a point $r_{0}=1+\epsilon$ close enough to the horizon $\epsilon \ll 1$ and to solve the second order ordinary differential Eq. (66) numerically toward the far-field region. In this region we easily can read off the coefficients $Y_{\text {in }}, Y_{\text {out }}$ by the $\chi^{2}$ fit, whose ratio directly leads to the greybody factor $\Gamma=1-$ $\left|Y_{\text {out }} / Y_{\text {in }}\right|^{2}$.

Putting the purely ingoing boundary condition $W_{\text {in }}=0$ at the near-horizon $r=r_{0}$ is always polluted by a tiny outgoing wave, numerically. This itself is the case for the scalar too. The difficulty in the spinor $(s=1 / 2)$ and vector $(s=1)$ case is that the amplitude of the outgoing wave grows with respect to that of the ingoing one as we go apart from $r_{0}$. To evade this problem, we first expand the nearhorizon solution

$$
\begin{aligned}
& R_{\mathrm{in}}^{\mathrm{NH}}=1+a_{1}(r-1)+a_{2}(r-1)^{2}+\cdots, \\
& R_{\mathrm{out}}^{\mathrm{NH}}=e^{2 i k r_{*}}(r-1)^{s}\left(1+b_{1}(r-1)+\cdots\right),
\end{aligned}
$$

where the coefficients $a_{i}, b_{i}$ are straightforwardly obtained by substituting the expansion of $\Delta, \eta, \zeta$ and (70) into (66), which serves linear equations for the coefficients. The point to remove the outgoing contamination is the following subtraction

$$
\tilde{R}=R-\left(1+a_{1}(r-1)\right),
$$

introduced in [35] as Bardeen's prescription. Then $\tilde{R}$ satisfies the equation

$$
\mathcal{L} \tilde{R}=g \text {, }
$$

where

$$
\begin{aligned}
& \mathcal{L}=d^{2} / d r^{2}+\eta d / d r+\zeta, \\
& g=-\mathcal{L}\left(1+a_{1}\left(r-r_{H}\right)\right)=-\eta a_{1}-\zeta\left(1+a_{1}\left(r-r_{H}\right)\right) .
\end{aligned}
$$

Now (72) can be safely solved toward the far-field region without the growing outgoing contamination.

\section{B. Matching at far field}

We expand the far-field solutions for spinor $(s=1 / 2)$

$$
\begin{aligned}
& R_{\mathrm{in}}^{\mathrm{FF}}=1+\frac{c_{1}^{f}}{r}+\frac{c_{2}^{f}}{r^{2}}+\frac{c_{3}^{f}}{r^{3}}+\cdots, \\
& R_{\mathrm{out}}^{\mathrm{FF}}=e^{2 i k r_{*}} \frac{1}{r}\left(1+\frac{d_{1}^{f}}{r}+\frac{d_{2}^{f}}{r^{2}} \cdots\right),
\end{aligned}
$$

and for vector $(s=1)$

$$
\begin{aligned}
& R_{\mathrm{in}}^{\mathrm{FF}}=r\left(1+\frac{c_{1}^{v}}{r}+\frac{c_{2}^{v}}{r^{2}}+\frac{c_{3}^{v}}{r^{3}}+\cdots\right), \\
& R_{\mathrm{out}}^{\mathrm{FF}}=e^{2 i k r_{*}} \frac{1}{r}\left(1+\frac{d_{1}^{v}}{r}+\frac{d_{2}^{v}}{r^{2}}+\frac{d_{3}^{v}}{r^{3}}+\cdots\right) .
\end{aligned}
$$

Again the coefficients $c_{i}^{f, v}, d_{i}^{f, v}$ can be straightforwardly obtained. The asymptotic form of the $\tilde{R}_{s}$ is now

$$
\begin{aligned}
\tilde{R}_{1 / 2}= & \left(Y_{\text {in }}+1-a_{1}\right)+a_{1} r+Y_{\text {in }} \frac{c_{1}^{f}}{r}+Y_{\text {out }} \frac{e^{2 i \omega r_{*}}}{r}, \\
\tilde{R}_{1}= & \left(Y_{\text {in }}-a_{1}\right) r+\left(Y_{\text {in }} c_{1}^{v}-1+a_{1}\right)+Y_{\text {in }} \frac{c_{2}^{v}}{r} \\
& +Y_{\text {out }} \frac{e^{2 i \omega r_{*}}}{r} .
\end{aligned}
$$

By the $\chi^{2}$ fit to the obtained solution $\tilde{R}$ in the far-field region, we can determine $Y_{\text {in }}, Y_{\text {out }}$ since the bigger terms than that containing $Y_{\text {out }}$ are all fixed. The smallness of the oscillating term containing $Y_{\text {out }}$ can be overcome easily by keeping sufficient digits in the numerics and by taking sufficiently dense sample points in the far-field region for the $\chi^{2}$ fit.

Finally the greybody factors can be obtained as

$$
\begin{gathered}
\Gamma_{s=1 / 2}=1-\frac{2 \omega}{\left|c_{1}^{f}\right|}\left|\frac{Y_{\text {out }}}{Y_{\text {in }}}\right|^{2}, \\
\Gamma_{s=1}=1-\frac{2 \omega^{2}}{\left|c_{2}^{v}\right|}\left|\frac{Y_{\text {out }}}{Y_{\text {in }}}\right|^{2} .
\end{gathered}
$$

\section{GREYBODY FACTORS AND HAWKING RADIATION SPECTRA}

In [25] we have argued that the black hole production cross section for a center of mass energy $s$ is well approximated by 


$$
\frac{d \sigma}{d J}(s)= \begin{cases}8 \pi J / s & \text { for } J<J_{\max }, \\ 0 & \text { for } J>J_{\max }\end{cases}
$$

except for the region where $J$ is very close to $J_{\max }$, and consequently that the black hole tends to be produced with large angular momentum. The rotation parameter $a$ corresponding to $J_{\max }$ is [25]

$$
a_{\max }=\frac{n}{2}+1
$$

for a $D=4+n$-dimensional black hole.

Once produced, we assume that the decay process of the black hole is governed by the Hawking radiation into the brane-localized standard model fields (see [51] for a review on the estimation of the amount of energy radiated at the black hole formation process (balding phase) rather than by the Hawking radiation and see also [52] for the recent progress) in the spirit that the quantum gravitational correction will be read off as a deviation from this precise prediction in the black hole picture.

With this assumption in mind, the number of spin $s$ particles emitted into a $(l, m)$ angular mode of the spheroidal harmonics is given, per degree of freedom per unit time per energy interval $[\omega, \omega+d \omega]$, by

$$
\frac{d N_{s, l, m}}{d \omega d t}=\frac{1}{2 \pi} \frac{\Gamma_{s, l, m}(\omega)}{e^{(\omega-m \Omega) / T}-(-1)^{2 s}},
$$

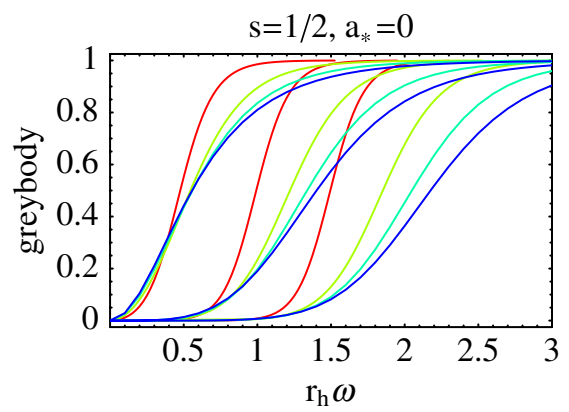

where $T=\left[(n+1)+(n-1) a_{*}^{2}\right] / 4 \pi r_{h}\left(1+a_{*}^{2}\right)$ and $\Omega=$ $a_{*} /\left(1+a_{*}^{2}\right) r_{h}$ are the Hawking temperature and angular velocity of the black hole, respectively. The corresponding power and angular spectra are obtained by multiplying the number spectrum (82) by $\omega$ and $m$, respectively.

The time evolution of the black hole with mass $M$ and angular momentum $J$ is then governed by

$$
-\frac{d}{d t}\left(\begin{array}{c}
M \\
J
\end{array}\right)=\sum_{s, l, m} g_{s} \int_{0}^{\infty} d \omega \frac{d N_{s, l, m}}{d \omega d t}\left(\begin{array}{c}
\omega \\
m
\end{array}\right),
$$

where $g_{s}$ is the number of massless degrees of freedom at temperature $T$, namely, the number of degrees of freedom whose masses are smaller than $T$ with spin $s$.

\section{A. Greybody factors for spinor and vector fields}

We present the greybody factors obtained by the procedure above. Hereafter we limit ourselves to the case $D \leq$ 11 motivated by the fact that $D=11$ is the highest possible dimension if we exclude the $s>2$ component fields from the supergravity multiplet, though in principle we can consider a larger-dimensional case as well [53].

In Fig. 1, we plot the greybody factors for the branelocalized spinor field for the nonrotating $\left(a_{*}=0\right)$ and highly rotating $\left(a_{*}=1.5\right)$ black holes. Note that $a_{*}=$ 1.5 is the highest possible rotation parameter for a $D=5$

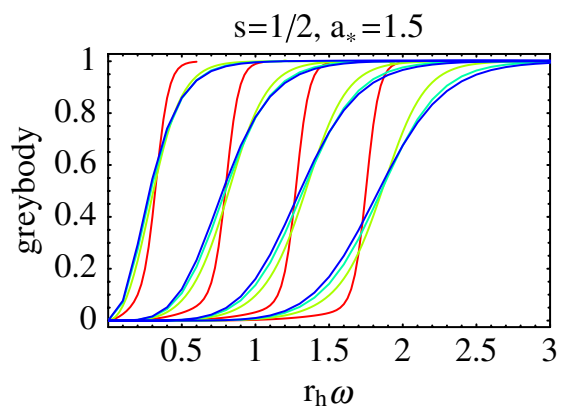

FIG. 1 (color online). Greybody factors for spinor field $(s=1 / 2)$ with fixed $a_{*}=0$ (left) and 1.5 (right). Each plot is for $l=m=$ $1 / 2,3 / 2,5 / 2$ modes (and 7/2 for right plot) from left to right bunches, respectively, and each bunch contains $D=5,7,9$, and 11 cases where the steeper curve corresponds to lower dimensions.
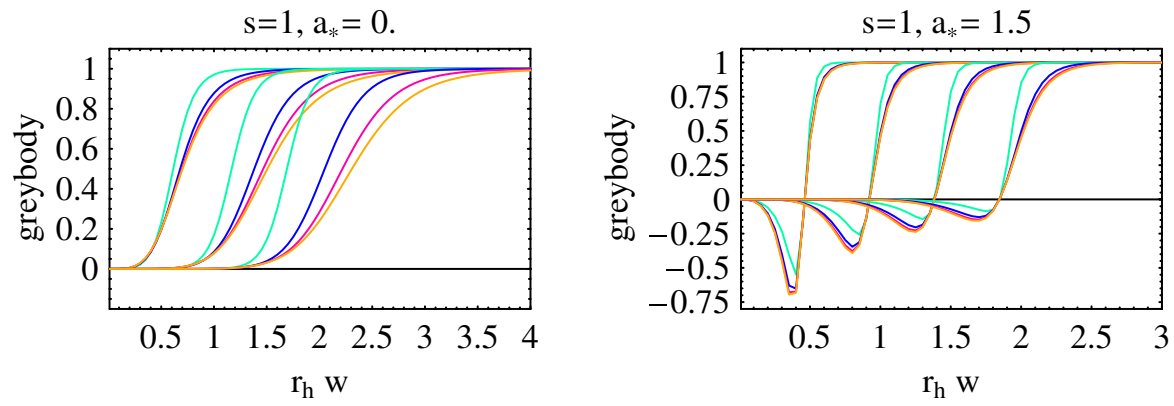

FIG. 2 (color online). Greybody factors for vector field $(s=1)$ with fixed $a_{*}=0$ (left) and 1.5 (right). Each plot is for $l=m=1,2$, 3 modes (and 4 for right plot) from left to right bunches, respectively, and each bunch contains $D=5,7,9$, and 11 cases where the steeper curve corresponds to lower dimensions. 

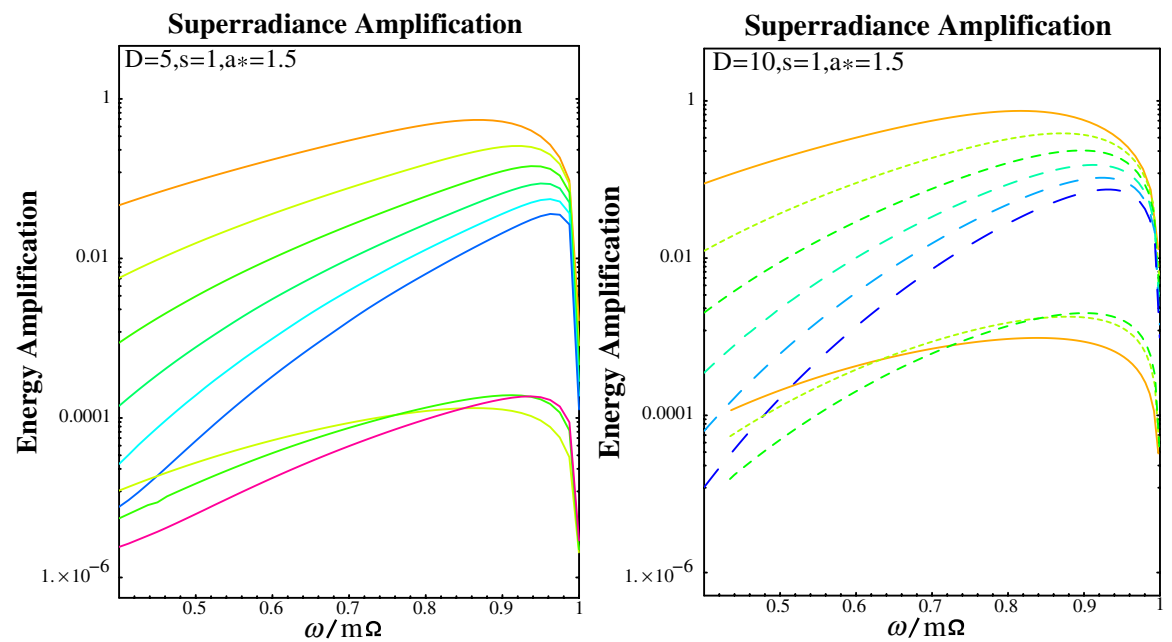

FIG. 3 (color online). Superradiant amplification of brane-localized vector field amplitude scattered by a highly rotating $\left(a_{*}=1.5\right)$ black hole in $D=5$ (left) and 10 (right).
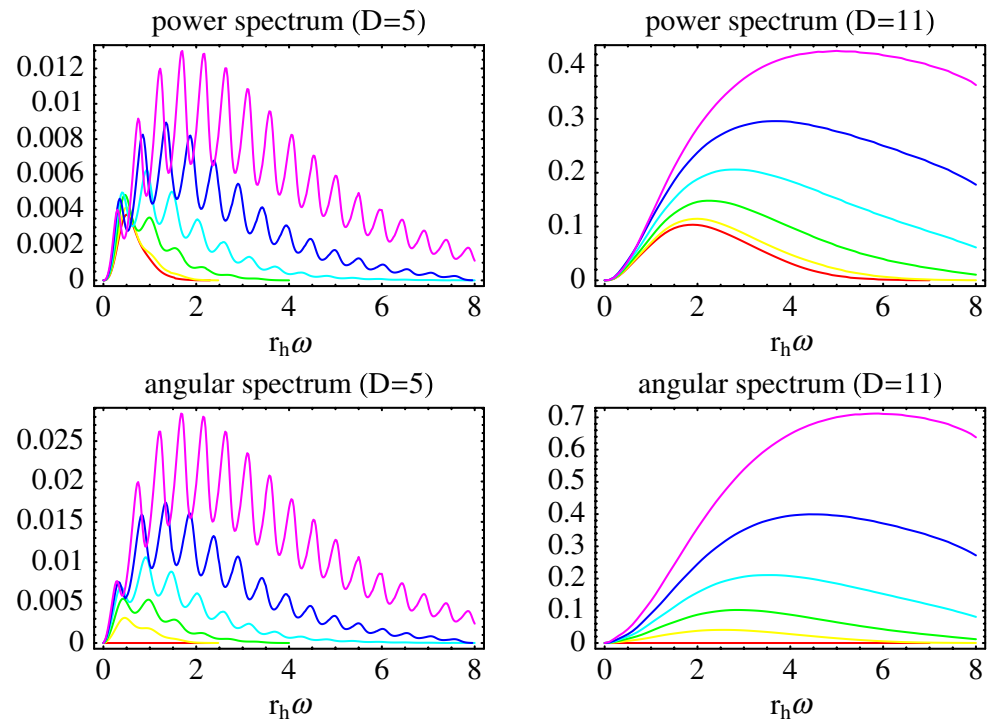

FIG. 4 (color online). Spinor power (upper) and angular (lower) spectra for fixed $D=5$ (left) and $D=11$ (right). In each figure, $a_{*}=0,0.3, \ldots, 1.5$ from lower left to upper right.
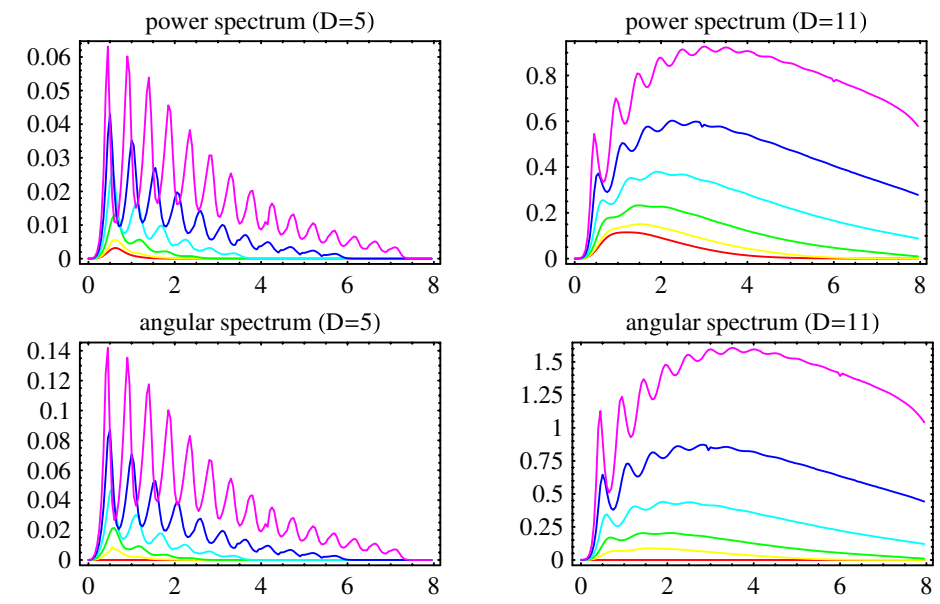

FIG. 5 (color online). Vector power (upper) and angular (lower) spectra for fixed $D=5$ (left) and $D=11$ (right). In each figure, $a_{*}=0,0.3, \ldots, 1.5$ from lower left to upper right. 

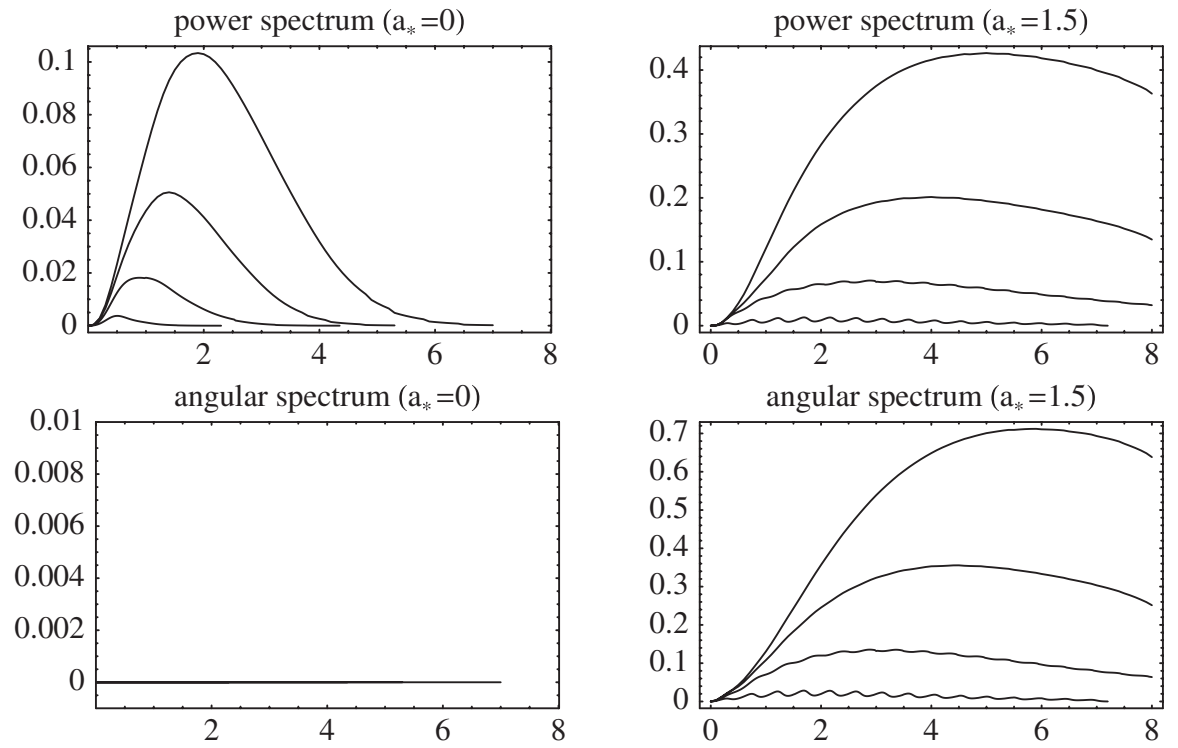

FIG. 6. Spinor power (upper) and angular (lower) spectra for fixed $a_{*}=0$ (left) and 1.5 (right). In each figure, $D=5,7,9$, and 11 from below to above.

black hole when the black hole is produced by a collision of two particles; see Eq. (81).

Figure 2 shows the corresponding plots for the vector field $(s=1)$. We observe that the greybody factor becomes negative when $\omega<m \Omega$, rendering the number spectrum (82) always positive. In other words, when the black hole is highly rotating, an incoming steady energy flux with $\omega<$ $m \Omega$ is scattered back by the hole with an increased amplitude; see Eq. (1). This is called the superradiance [54]. Though we cannot observe the superradiance itself for the $\mathrm{TeV}$ black hole since it is decaying so fast, we show the rate of the energy amplification in Fig. 3 for its own interest.
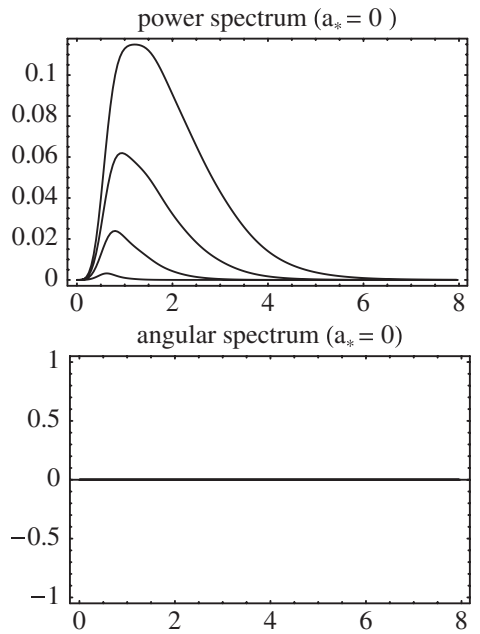

\section{B. Power and angular spectra for spinor}

Hereafter in this section, we present the dimensionless power and angular spectra per degree of freedom:

$$
\begin{aligned}
r_{h} \frac{d E_{s, l, m}}{d \omega d t} & =\frac{1}{2 \pi} \frac{r_{h} \omega \Gamma_{s, l, m}(\omega)}{e^{(\omega-m \Omega) / T}-(-1)^{2 s}}, \\
\frac{d J_{s, l, m}}{d \omega d t} & =\frac{1}{2 \pi} \frac{m \Gamma_{s, l, m}(\omega)}{e^{(\omega-m \Omega) / T}-(-1)^{2 s}},
\end{aligned}
$$

versus the dimensionless energy of the emitted particle $r_{h} \omega$. Note that this implies that the frequency $\omega$ in the horizontal axis is given in units of $r_{h}^{-1}$, which varies for a fixed mass $M$ when we vary the angular momentum $J$. For
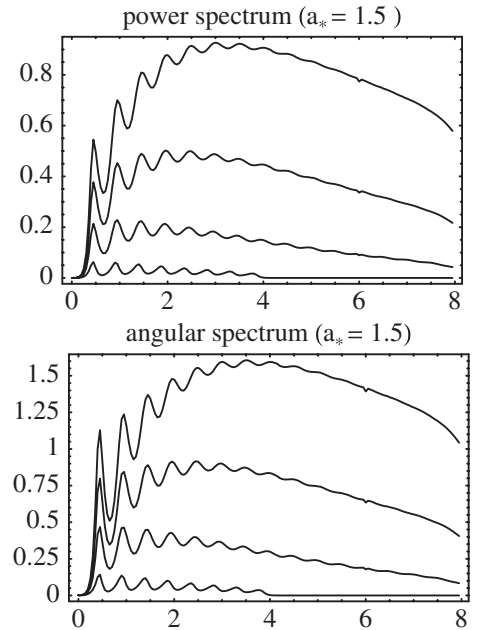

FIG. 7. Vector power (upper) and angular (lower) spectra for fixed $a_{*}=0$ (left) and 1.5 (right). In each figure, $D=5,7,9$, and 11 from below to above. 

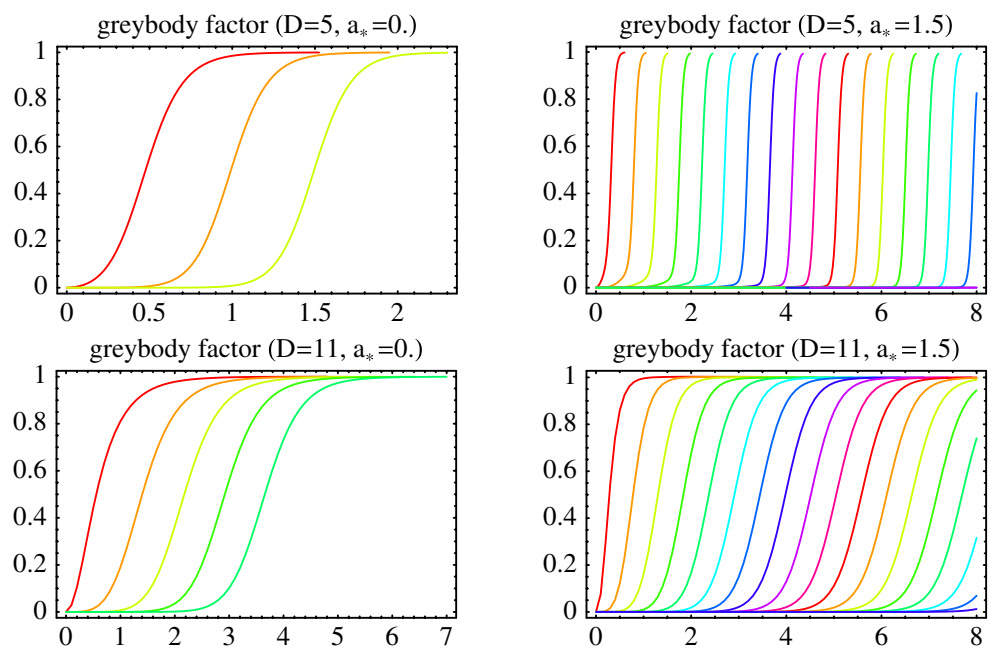

FIG. 8 (color online). Spinor greybody factor for $\left(D, a_{*}\right)=(5,0),(5,1.5),(11,0)$ and $(11,1.5)$. The contributions from the angular modes with the same $l$ mod 10 are drawn with the same color/gray level.
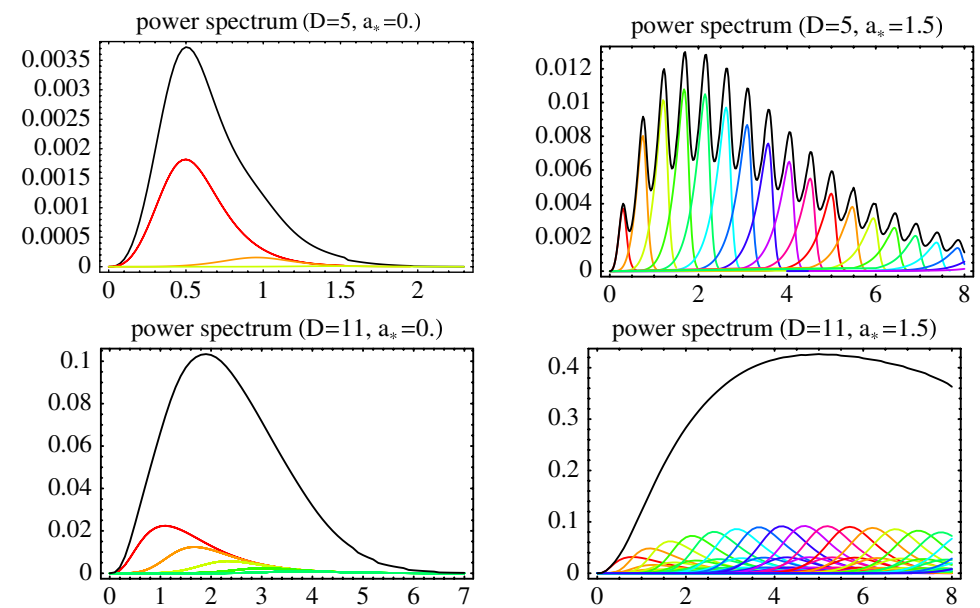

FIG. 9 (color online). Spinor power spectra for $\left(D, a_{*}\right)=(5,0),(5,1.5),(11,0)$ and $(11,1.5)$. The contributions from the angular modes with the same $l$ mod 10 are drawn with the same color/gray level. In each figure, the uppermost enveloping black curve is the total spectrum.
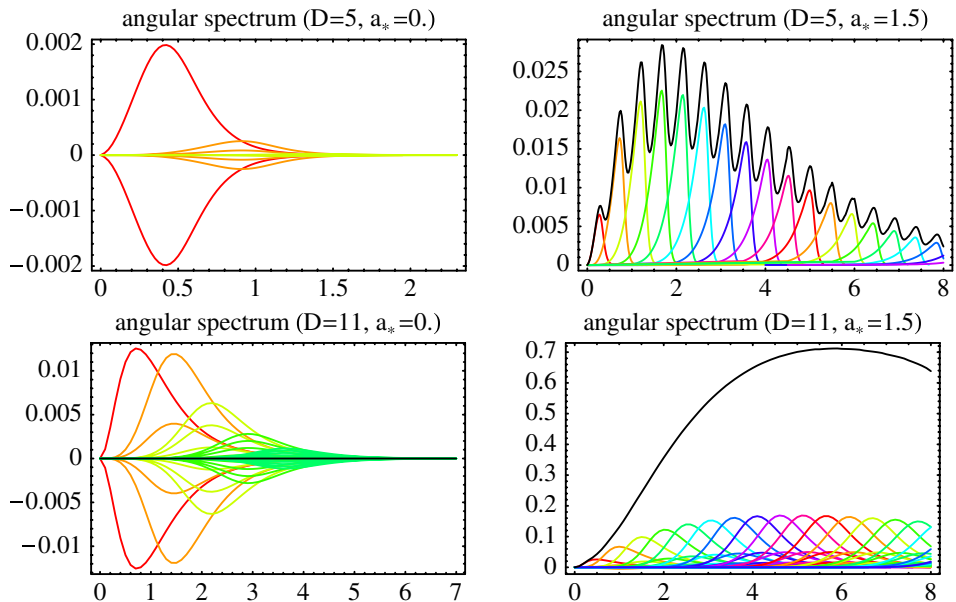

FIG. 10 (color online). Spinor angular spectra for $\left(D, a_{*}\right)=(5,0),(5,1.5),(11,0)$ and $(11,1.5)$. The contributions from the angular modes with the same $l$ mod 10 are drawn with the same color/gray level. In each figure with $a_{*}=1.5$, the uppermost enveloping black curve is the total spectrum. 

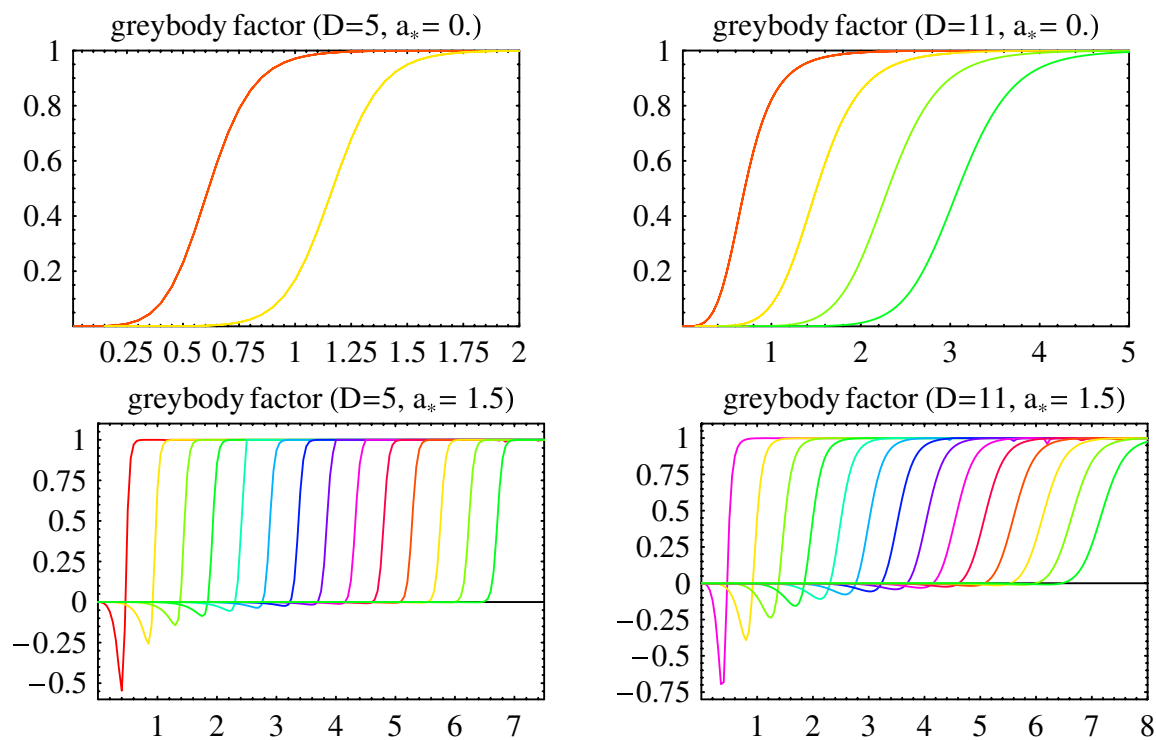

FIG. 11 (color online). Vector greybody factor for $\left(D, a_{*}\right)=(5,0),(5,1.5),(11,0)$ and $(11,1.5)$. The contributions from the angular modes with the same $l$ mod 10 are drawn with the same color/gray level.

simpler presentation we show our plot for $0<r_{h} \omega<8$ in this section but we also have calculated all the $\omega$ regions for $a_{*} \leq 1.5$ to obtain the total power and angular spectra in the next section; see also Figs. 14.

In Figs. 4 and 5, we plot them for the brane-localized spinor and vector fields, respectively, with varying black hole rotation $a_{*}$ for fixed $D=5$ (Randall-Sundrum black hole) and $D=11$ (ADD black hole). It is clear that the larger the rotation parameter $a_{*}$ is, the more enhanced are both the power and angular spectra.

In Figs. 6 and 7, we plot the same for spinor and vector, respectively, for varying dimensions $D$ with fixed black hole rotation $a_{*}=0$ (nonrotating) and $a_{*}=1.5$ (highly
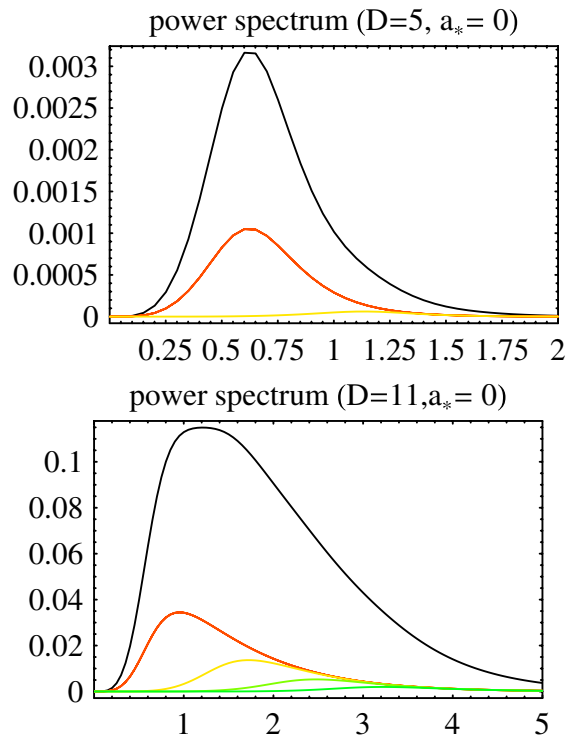

rotating). These figures show that the black hole radiation is greatly enhanced for larger dimensions. Note that the peaks coming from $l=m$ modes are distinctive only for the Randall-Sundrum black hole, especially in the case of the spinorial emission.

The substructure behind the total spectrum is shown in Figs. 8-10 and in Figs. 11-13. For each column, the greybody factor, power spectrum, and the angular spectrum are shown from above to below. We can see that each region of the greybody factor's rise in $r_{h} \omega$ coincides with each peak. For a nonrotating hole, the angular modes $m=-l,-l+$ $1, \ldots, l$ give exactly the same greybody factors and the $\pm m$ modes cancel each other in each given $l$ mode for angular
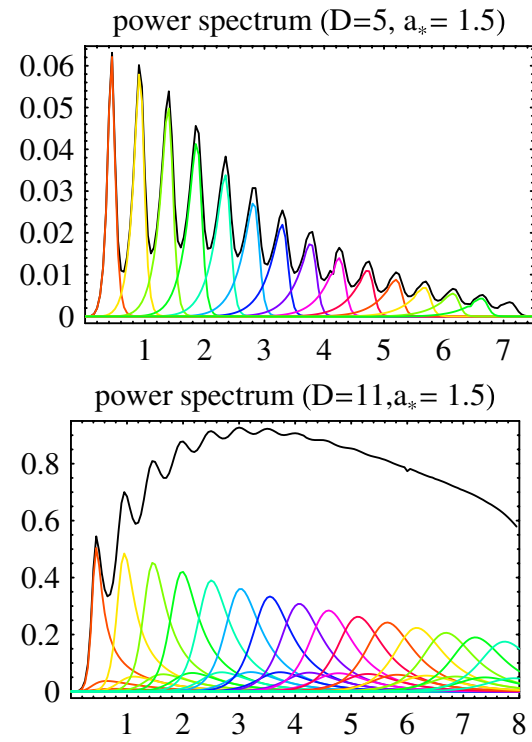

FIG. 12 (color online). Vector power spectra for $\left(D, a_{*}\right)=(5,0),(5,1.5),(11,0)$ and $(11,1.5)$. The contributions from the angular modes with the same $l$ mod 10 are drawn with the same color/gray level. In each figure, the uppermost enveloping black curve is the total spectrum. 

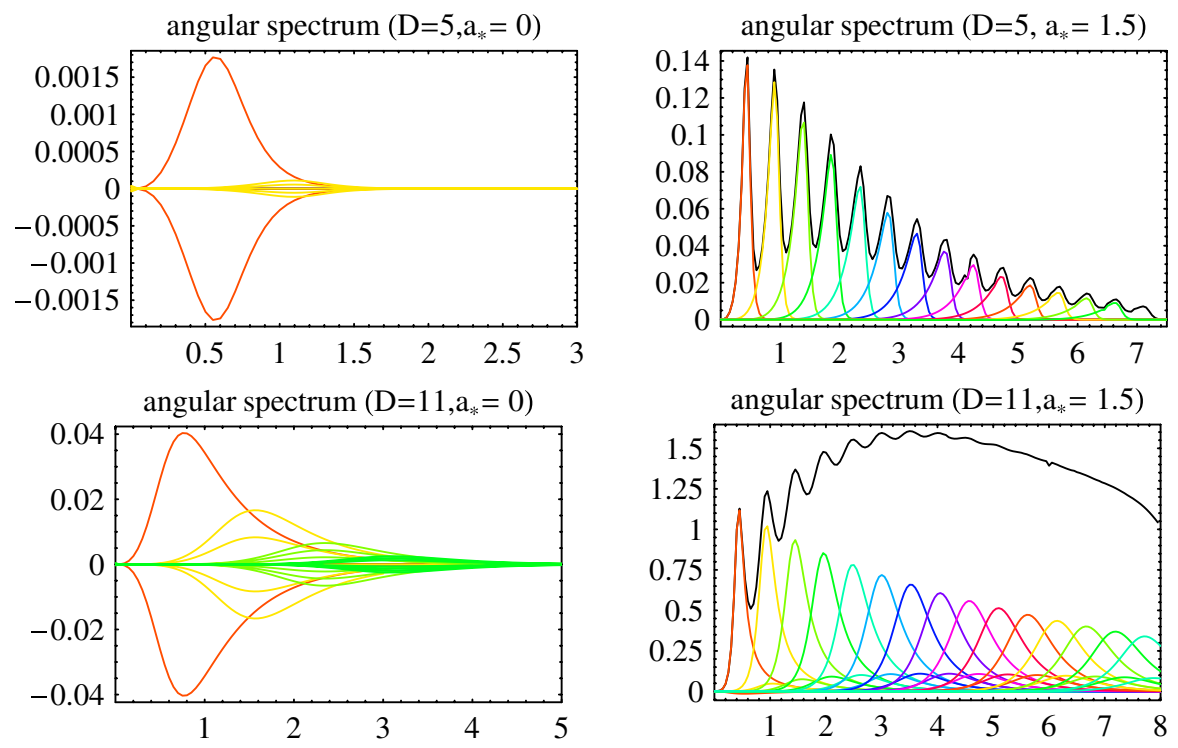

FIG. 13 (color online). Vector angular spectra for $\left(D, a_{*}\right)=(5,0),(5,1.5),(11,0)$ and $(11,1.5)$. The contributions from the angular modes with the same $l \bmod 10$ are drawn with the same color/gray level. In each figure with $a_{*}=1.5$, the uppermost enveloping black curve is the total spectrum.
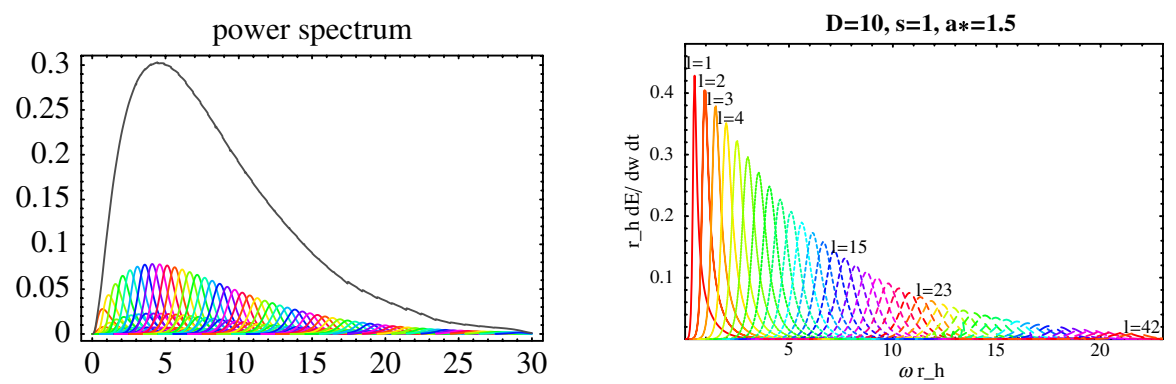

FIG. 14 (color online). Power spectrum from a $D=10$-dimensional black hole for spinor (left) and vector (right). Left: similarly plotted as in Figs. 8-10. Right: contributions of $l=m$ modes are shown.

spectrum. When the black hole is highly rotating, the contributions from the $l=m$ modes become dominant.

For a highly rotating black hole with large $a_{*}$, the contributions from $l=m$ modes become dominant, which greatly enhance the amplitude of total spectra at high frequency region $r_{h} \omega>1$. This is sometimes called the superradiant enhancement of the higher spin emission, though it is not directly related to the original meaning [54] of the superradiance as shown in Fig. 3. To exhibit such high frequency tails which are taken into account in the calculation of the next section, we present Fig. 14.

\section{TIME EVOLUTION OF A BLACK HOLE}

In Fig. 15, we show the schematic pictures for the black hole life at various stages: production phase, the balding phase, the spin-down phase, the Schwarzschild phase, and the Planck phase [23]. The spin-down and Schwarzschild phases are of interest here.

We show the black hole time evolution as it emits branelocalized particles. We calculate the rates at which energy and angular momentum are radiated into the brane-

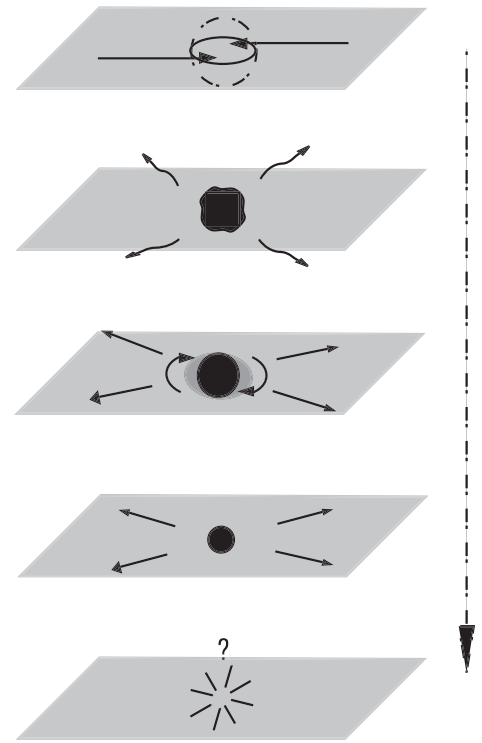

FIG. 15. Schematic pictures for black hole life. From above to below, the production phase, the balding phase, spin-down phase, Schwarzschild phase, and Planck phase are shown. 
localized standard model fields and the evolution of the mass and the angular momentum. A particular quantity of interest is the portion of the energy emitted during the spindown phase and the (almost) Schwarzschild phase.

\section{A. Setup for time evolution}

To follow the time evolution, it is convenient to make quantities invariant under the scaling of $r_{s}=(1+$ $\left.a_{*}^{2}\right)^{1 /(n+1)} r_{h}$, which does not vary for a fixed $M$ when we vary $J$. We define a scale-invariant function $\gamma\left(a_{s}\right)$, with respect to the scale-invariant rotation parameter $a_{s}=a / r_{s}$, as follows:

$$
\begin{gathered}
\gamma^{-1}\left(a_{s}\right) \equiv \frac{d \ln a_{s}}{d \ln M / M_{i}} \\
=\frac{n+2}{2}\left(\frac{1}{a} \frac{d J}{d M}-\frac{2}{n+1}\right),
\end{gathered}
$$

where the mass of a hole is measured in the unit of the initial mass $M_{i}$. This quantity is directly integrated. We calculate the ratio of the final mass $M_{f}$ to the initial mass $M_{i}$ by integrating Eq. (85) with an initial rotation parameter $a_{s}$ (ini)

$$
\frac{M_{f}}{M_{i}}=\exp \left(\int_{a_{s}(\text { ini })}^{a_{s}(\text { final })} d a_{s} \frac{\gamma\left(a_{s}\right)}{a_{s}}\right) .
$$

The amount of energy which is radiated in spin-down phase $\left[0 \approx a_{s}\right.$ (final) $\left.\leq a_{s} \leq a_{s}(\mathrm{ini})\right]$ is $\left(M_{i}-M_{f}\right)$ and then the remaining $M_{f}$ will be subsequently radiated off in the Schwarzschild phase, where the angular momentum of black hole is vanishing.

Next, we consider the evolution of the black hole. Since the time roughly scales as $r_{s}^{n+3}$ in $(4+n)$ dimensions, it is convenient to introduce scale-invariant rates for energy and angular momentum as follows ${ }^{2}$ :

$$
\begin{gathered}
\alpha\left(a_{s}\right) \equiv-r_{s}^{n+3} \frac{d \ln M}{d t}, \\
\beta\left(a_{s}\right) \equiv-r_{s}^{n+3} \frac{d \ln J}{d t} .
\end{gathered}
$$

With these new variables $\gamma\left(a_{s}\right)$ can be written as $\gamma^{-1}\left(a_{s}\right)=\beta / \alpha\left(a_{s}\right)-(n+2) /(n+1)$. For all the standard model particles,

$$
\begin{aligned}
& \alpha_{\mathrm{SM}}=g_{s} \alpha_{s=0}+g_{f} \alpha_{s=1 / 2}+g_{v} \alpha_{s=1}, \\
& \beta_{\mathrm{SM}}=g_{s} \beta_{s=0}+g_{f} \beta_{s=1 / 2}+g_{v} \beta_{s=1},
\end{aligned}
$$

\footnotetext{
${ }^{2} \mathrm{We}$ can understand this by simply looking at the formula $-d M / d t \sim A T^{4}$ where the surface area of horizon $A \sim r_{s}^{2}$ for brane fields and the temperature of the hole $T \sim 1 / r_{s}$ and $M \sim$ $r_{s}^{n+1}$.
}

where $g_{s}=4, g_{f}=90$, and $g_{v}=24$ are adopted in this section.

We also introduce dimensionless variables $y$ and $z$ to take angular momentum and mass of the hole:

$$
\begin{aligned}
y & \equiv-\ln a_{s}, \\
z & \equiv-\ln \frac{M}{M_{i}},
\end{aligned}
$$

then finally we get the time variation of energy and angular momentum in terms of scale-invariant time parameter $[\tau=$ $r_{s}^{-n-3}$ (ini) $\left.t\right]$ with initial mass of the hole:

$$
\begin{aligned}
& \frac{d z}{d y}=\frac{\alpha}{\beta-\alpha\left(\frac{n+2}{n+1}\right)} \\
& \frac{d y}{d \tau}=\left(\beta-\alpha\left(\frac{n+2}{n+1}\right)\right) e^{[(n+3) /(n+1)] z} .
\end{aligned}
$$

After finding the solutions $z(y)$ and $\tau(y)$ of the coupled differential Eqs. (94), one can get $y(\tau)$ and $z(\tau)$, hence $a_{s}$ and $M / M_{i}$, as a function of time. From these, one can find how other quantities evolve, such as the area.

For our purpose, it is convenient to convert the set of variables $\left(r_{h}, a_{*}\right)$. For conversion of unit, the following expressions are useful with $a_{s}=a_{*} /\left(1+a_{*}^{2}\right)^{1 /(n+1)}$ :

$$
\begin{gathered}
\alpha\left(a_{s}\right)=-\iota_{n}^{n+1}\left(1+a_{*}^{2}\right)^{2 /(n+1)} r_{h}^{2} \frac{d M}{d t}, \\
\beta\left(a_{s}\right)=-\kappa_{n}^{n+1}\left(1+a_{*}^{2}\right)^{2 /(n+1)} r_{h}^{2} \frac{1}{a} \frac{d J}{d t},
\end{gathered}
$$

where

$$
\begin{gathered}
\iota_{n}=r_{s} M^{-1 /(n+1)}=\left(\frac{16 \pi G}{(n+2) \Omega_{n+2}}\right)^{1 /(n+1)}, \\
\kappa_{n}=\iota_{n}\left(\frac{n+2}{2}\right)^{1 /(n+1)} .
\end{gathered}
$$

\section{B. Results for time evolution}

In this section we use the natural unit $8 \pi G=1$. We assume that all the standard model fields are massless and therefore the effective degrees of freedom are given by $g_{s}=4, g_{f}=90$, and $g_{v}=24$. In Fig. 16, we draw the rates at which the energy and angular momentum are radiated into the brane-localized standard model fields. We have explicitly calculated the rates up to the rotation parameter $a_{*}<1.5$ for the Randall-Sundrum black hole $(D=5)$ and for the ADD black hole $(D=10)$. For $D=$ 10 we have extrapolated our result using the cubic-curve approximation up to $a_{*}<4$ to cover all the possible rotations; see Eq. (81). The curves are plotted with respect to $a_{s}$ rather than $a_{*}$ using the conversion rules described in the previous section, namely, up to $a_{s}<0.83$ and 2.67 for $D=$ 5 and 10 , respectively. The $D=10$ results are exact without using the cubic-curve extrapolation up to $a_{s}<1.27$. 

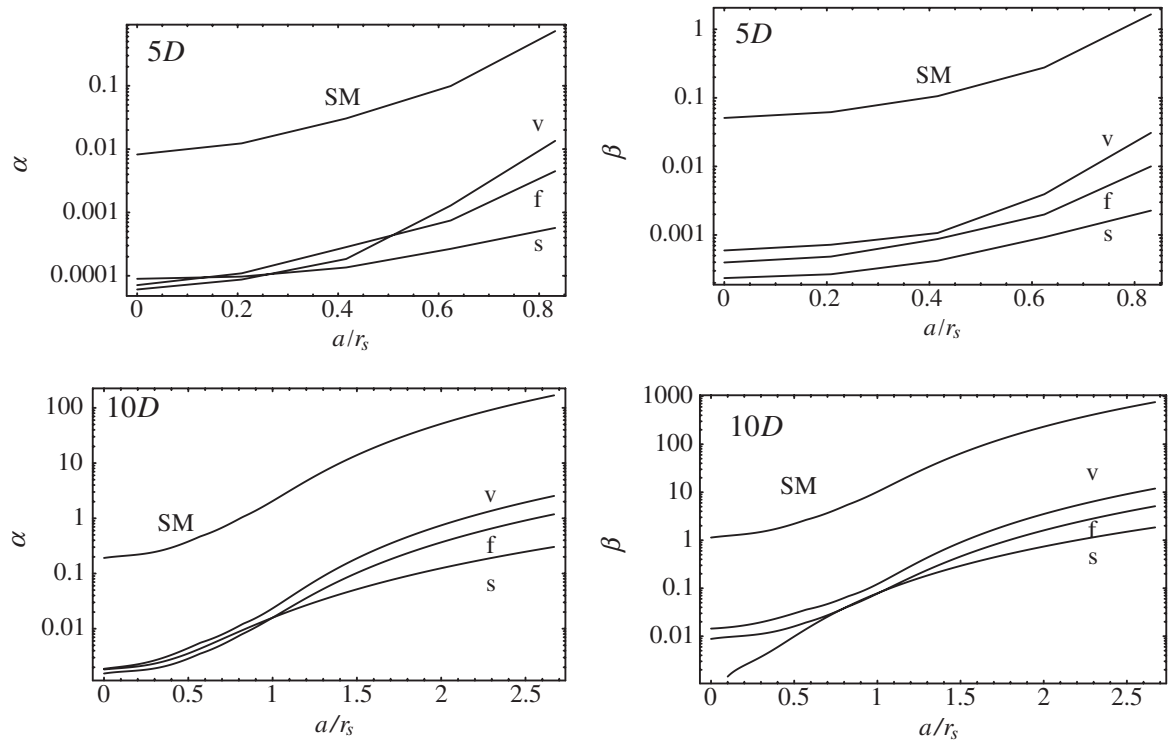

FIG. 16. Total mass loss rate $\alpha$ (left) and angular momentum loss rate $\beta$ (right) for scalar $(s)$, fermion $(f)$, vector $(v)$, and for sum of all the standard model particles (SM) in $D=5$ (above) and 10 (below).

In Fig. 16, one can see that the power $\alpha$ and torque $\beta$ are increasing functions of the angular momentum $a_{s}$. The vector emission dominates over the spinor and scalar emission for the high rotation parameter, sometimes called the superradiant enhancement of the higher spin particle emission, but as rotation becomes slower the fermion channel becomes increasingly important. Generally, angular momentum is emitted much faster than energy, therefore a rapidly rotating black hole spins down to a nearly nonrotating state before its mass has been radiated off completely.

In Fig. 17, we plot the mass of a hole as a function of the rotation $a_{s}$ for the virtual setup where only the scalar $(s)$, spinor $(f)$, or vector $(v)$ field is emitted, respectively, and for the realistic case where all the standard model fields are emitted (SM). One can see that the larger the particle's spin is, the more effectively the black hole angular momentum is carried away. For the most effective case of vector-only $(v)$, the angular momentum is carried away so rapidly that more than $30 \%$ of the mass still remains after the spindown phase, to be radiated off at the Schwarzschild phase. In contrast, the scalar-only case $(s)$ exhibits that the black

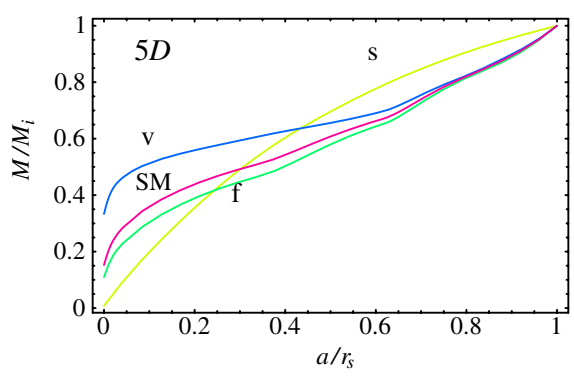

hole radiates its whole mass before it stops rotation. For the realistic case (SM), black hole loses roughly $70 \%$ to $80 \%$ in $D=5$ and $D=10$, respectively, before it stops rotation when starting from the maximum rotation. Note that the scalar emission $(s)$ is subdominant comparing to the vector $(v)$ and spinors $(f)$ because of its small effective degrees of freedom and small emission rates.

In Figs. 18 and 19, we plot the time evolution of rotation parameter and mass. The unit time $t_{0}$ is defined by the time duration from the initial state to the state with $a_{s}=0.01$ for the virtual cases of spinor-only $(f)$ and vector-only $(v)$. For scalar-only case $(s)$, the mass goes to zero before rotation stops, therefore we defined $t_{0}$ for scalar to be the duration until the whole mass is radiated off. The initial rotation parameter is fixed by $a_{s}=0.83$ and 2.67 in $D=5$ and $D=10$, respectively. The mass of the hole goes to zero before the rotation parameter goes to zero when only scalar emission is available. We have taken the initial radiation parameter to be $a_{s}=0.83$ and 2.67 for $D=5$ and 10 that are the maximal rotations allowed by the initial collision.

When all the standard model fields are turned on, the evolution is essentially determined by the spinor and vector

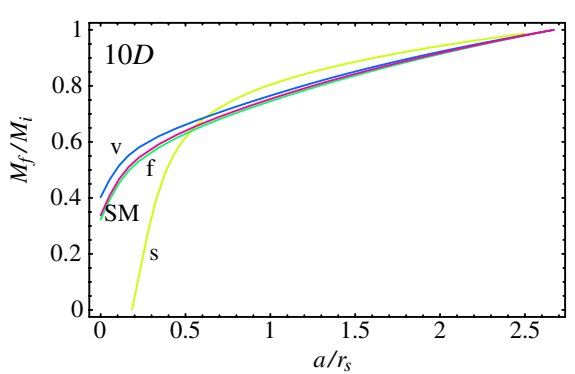

FIG. 17 (color online). Black hole mass evolution in units of the initial mass as a function of rotation parameter $a_{s}$ for scalar ( $s$ ), fermion $(f)$, vector $(v)$, and sum of all the standard model particles (SM) in $D=5$ (left) and $D=10$ (right). 

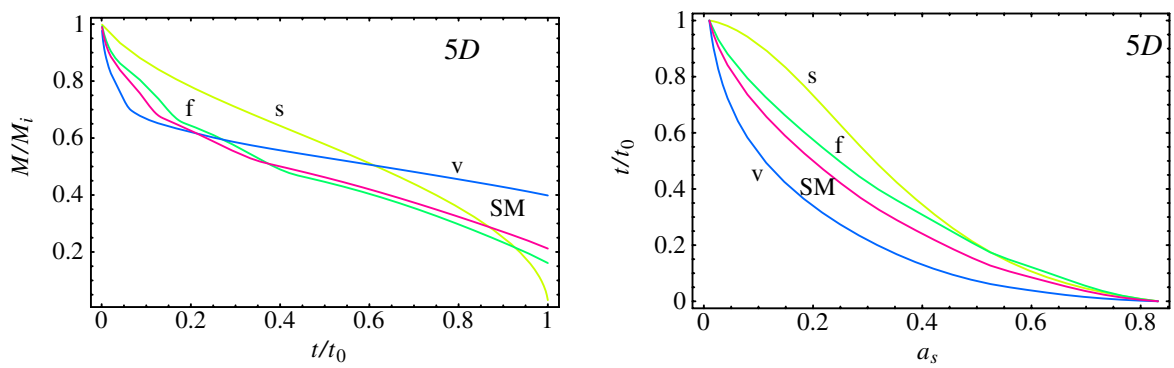

FIG. 18 (color online). Time evolution of mass and time versus rotation parameter in $D=5$.
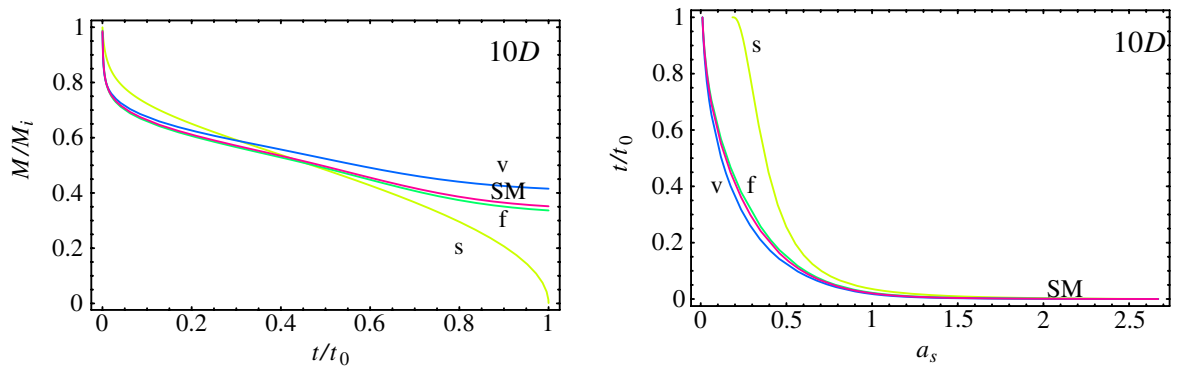

FIG. 19 (color online). Time evolution of mass and time versus rotation parameter in $D=10$.

radiation. The figures show that a black hole spins down quickly at the first stage with large rotation parameter and the decrease of rotation parameter slows down as angular momentum of the hole is reduced.

\section{SUMMARY AND DISCUSSION}

We have generalized our previous result [25] for the greybody factor and Hawking radiation spectrum of the higher-dimensional Kerr black hole to general dimensions $D=4+n$, without relying on the low frequency limit $r_{h} \omega \ll 1$, not only for the scalar field $(s=0)$ [40] but also for the spinor $(s=1 / 2)$ and vector fields $(s=1)$. Now we can completely describe the evolution of a black hole with any given initial mass and angular momentum by taking into account any type of field residing on the brane.

We have developed the numerical method to solve the radial Teukolsky equation which has been generalized to the higher dimension $(D=4+n)$ in the first paper [25] of our series. There are two points in our numerical methods. First, we have imposed the proper purely ingoing boundary condition near the horizon without the growing contamination of the outgoing wave by extracting lower order terms explicitly. Second, we have developed the method to fit the ingoing and outgoing part from the numerically integrated wave solution at a far-field region by explicitly obtaining the next-to-next order expansion (or next-tonext-to-next order in vector case) of the solution. With this progress in numerical treatment, we can safely integrate the generalized Teukolsky equation up to very large $r$ without outgoing wave contamination.

Then we have calculated all the possible modes to completely determine the radiation rate of the mass and angular momentum of the hole. Totally 3407 modes are computed explicitly, other than the modes which are confirmed to be negligible. A black hole tends to lose its angular momentum at the early stage of evolution. However, the black hole still has a sizable rotating parameter after radiating half of its mass. Typically more than $70 \%$ or $80 \%$ of a black hole's mass is lost during the spin-down phase. In the case of very fast initial rotation, the number could be modified quantitatively by taking the bulk graviton emission into account, especially for a larger number of extra dimensions as discussed in the introduction, but the result would remain the case qualitatively. ${ }^{3}$

We have determined the radiation and evolution of the spin-down and Schwarzschild phases up to the ambiguities for the initial fastest rotations shown above. The remaining hurdle is the evaluation of the balding phase, which is still being disputed due to its nonperturbative nature, to extract the quantum gravitational information at the Planck phase from the experimental data at the CERN Large Hadron Collider.

\section{ACKNOWLEDGMENTS}

We would like to thank K. Hagiwara, Y. Okada, V.S. Rychkov, S. A. Teukolsky, and H. Yoshino for useful discussion. K. O. is supported by EU Grant No. MRTN-CT2004-503369, and S.C.P. is supported by the Korea Research Foundation Grant funded by the Korean Government (MOEHRD), Grant No. KRF-2005-214C00147.

\footnotetext{
${ }^{3}$ We note that a friction between the brane and black hole can be sizable and also can reduce the angular momentum faster at the initial stage $[55,56]$.
} 
[1] J. D. Bekenstein, Phys. Rev. D 7, 2333 (1973).

[2] S. W. Hawking, Commun. Math. Phys. 43, 199 (1975).

[3] S. W. Hawking, Phys. Rev. D 13, 191 (1976).

[4] S. B. Giddings, hep-th/9508151.

[5] A. Strominger and C. Vafa, Phys. Lett. B 379, 99 (1996).

[6] G. T. Horowitz and J. Polchinski, Phys. Rev. D 55, 6189 (1997).

[7] T. Damour and G. Veneziano, Nucl. Phys. B568, 93 (2000).

[8] G. 't Hooft, Phys. Lett. B 198, 61 (1987).

[9] N. Arkani-Hamed, S. Dimopoulos, and G. R. Dvali, Phys. Lett. B 429, 263 (1998).

[10] I. Antoniadis, N. Arkani-Hamed, S. Dimopoulos, and G. R. Dvali, Phys. Lett. B 436, 257 (1998).

[11] N. Arkani-Hamed, S. Dimopoulos, and G. R. Dvali, Phys. Rev. D 59, 086004 (1999).

[12] L. Randall and R. Sundrum, Phys. Rev. Lett. 83, 3370 (1999).

[13] P. C. Argyres, S. Dimopoulos, and J. March-Russell, Phys. Lett. B 441, 96 (1998).

[14] T. Banks and W. Fischler, hep-th/9906038.

[15] R. Emparan, G. T. Horowitz, and R. C. Myers, Phys. Rev. Lett. 85, 499 (2000).

[16] V.P. Frolov and D. Stojkovic, Phys. Rev. D 66, 084002 (2002).

[17] V. P. Frolov and D. Stojkovic, Phys. Rev. Lett. 89, 151302 (2002).

[18] M. Cavaglia, Phys. Lett. B 569, 7 (2003).

[19] D. Stojkovic, Phys. Rev. Lett. 94, 011603 (2005).

[20] A. S. Cornell, W. Naylor, and M. Sasaki, J. High Energy Phys. 02 (2006) 012.

[21] V. Cardoso, M. Cavaglia, and L. Gualtieri, J. High Energy Phys. 02 (2006) 021.

[22] S. Creek, O. Efthimiou, P. Kanti, and K. Tamvakis, Phys. Lett. B 635, 39 (2006).

[23] S. B. Giddings and S. D. Thomas, Phys. Rev. D 65, 056010 (2002).

[24] S. Dimopoulos and G. Landsberg, Phys. Rev. Lett. 87, 161602 (2001).

[25] D. Ida, K. Oda, and S. C. Park, Phys. Rev. D 67, 064025 (2003).

[26] H. Yoshino and Y. Nambu, Phys. Rev. D 67, 024009 (2003).

[27] H. Yoshino and V. S. Rychkov, Phys. Rev. D 71, 104028 (2005).

[28] L. A. Anchordoqui, J. L. Feng, H. Goldberg, and A. D.
Shapere, Phys. Rev. D 68, 104025 (2003).

[29] S. C. Park and H. S. Song, J. Korean Phys. Soc. 43, 30 (2003).

[30] R. Penrose and W. Rindler, Spinors and Space-time (Cambridge University Press, Cambridge, England, 1984), Vol. 1.

[31] R. C. Myers and M. J. Perry, Ann. Phys. (N.Y.) 172, 304 (1986).

[32] P. Kanti and J. March-Russell, Phys. Rev. D 67, 104019 (2003).

[33] C. M. Harris and P. Kanti, J. High Energy Phys. 10 (2003) 014.

[34] S. A. Teukolsky, Astrophys. J. 185, 635 (1973).

[35] S. A. Teukolsky, Astrophys. J. 185, 649 (1973).

[36] S. A. Teukolsky, Astrophys. J. 193, 443 (1974).

[37] D. N. Page, Phys. Rev. D 13, 198 (1976).

[38] D. N. Page, Phys. Rev. D 14, 3260 (1976).

[39] D. Ida, K. Oda, and S. C. Park, hep-ph/0501210.

[40] D. Ida, K. Oda, and S. C. Park, Phys. Rev. D 71, 124039 (2005).

[41] C. M. Harris, hep-ph/0502005.

[42] C. M. Harris and P. Kanti, Phys. Lett. B 633, 106 (2006).

[43] M. Casals, P. Kanti, and E. Winstanley, J. High Energy Phys. 02 (2006) 051.

[44] G. W. Gibbons, D. Ida, and T. Shiromizu, Prog. Theor. Phys. Suppl. 148, 284 (2003).

[45] G. W. Gibbons, D. Ida, and T. Shiromizu, Phys. Rev. Lett. 89, 041101 (2002).

[46] G. W. Gibbons, D. Ida, and T. Shiromizu, Phys. Rev. D 66, 044010 (2002).

[47] Y. Morisawa and D. Ida, Phys. Rev. D 69, 124005 (2004).

[48] M. Rogatko, Classical Quantum Gravity 19, L151 (2002).

[49] M. Rogatko, Phys. Rev. D 67, 084025 (2003).

[50] E. Seidel, Classical Quantum Gravity 6, 1057 (1989).

[51] V. Cardoso, E. Berti, and M. Cavaglia, Classical Quantum Gravity 22, L61 (2005).

[52] H. Yoshino, T. Shiromizu, and M. Shibata, Phys. Rev. D 72, 084020 (2005)

[53] J. L. Hewett, B. Lillie, and T. G. Rizzo, Phys. Rev. Lett. 95, 261603 (2005).

[54] W. H. Press and S. A. Teukolsky, Nature (London) 238, 211 (1972).

[55] V.P. Frolov, D. V. Fursaev, and D. Stojkovic, J. High Energy Phys. 06 (2004) 057.

[56] V.P. Frolov, D. V. Fursaev, and D. Stojkovic, Classical Quantum Gravity 21, 3483 (2004). 